\begin{tabular}{|llll|}
\hline & \multicolumn{1}{c|}{ S A M P E } & I N F O R M A T I O N \\
\hline \hline Sample Name: & 13620-148 & Acquired By: & walkup \\
Sample Type: & Unknown & Sample Set Name: & thuy20190625 \\
Vial: & 1 & Acq. Method Set: & MethodSetAB5T95B15Min \\
Injection \#: & 1 & Processing Method: & 220W280W15MIN \\
Injection Volume: & $10.00 \mathrm{ul}$ & Channel Name: & W2996 280.0nm-4.0 \\
Run Time: & 30.0 Minutes & Proc. Chnl. Descr.: & W2996 PDA 280.0 nm at 4.0 \\
& & & \\
Date Acquired: & 6/25/2019 4:45:15 PM EDT & & \\
Date Processed: & $7 / 26 / 201912: 41: 28$ PM EDT & & \\
\hline
\end{tabular}

Auto-Scaled Chromatogram

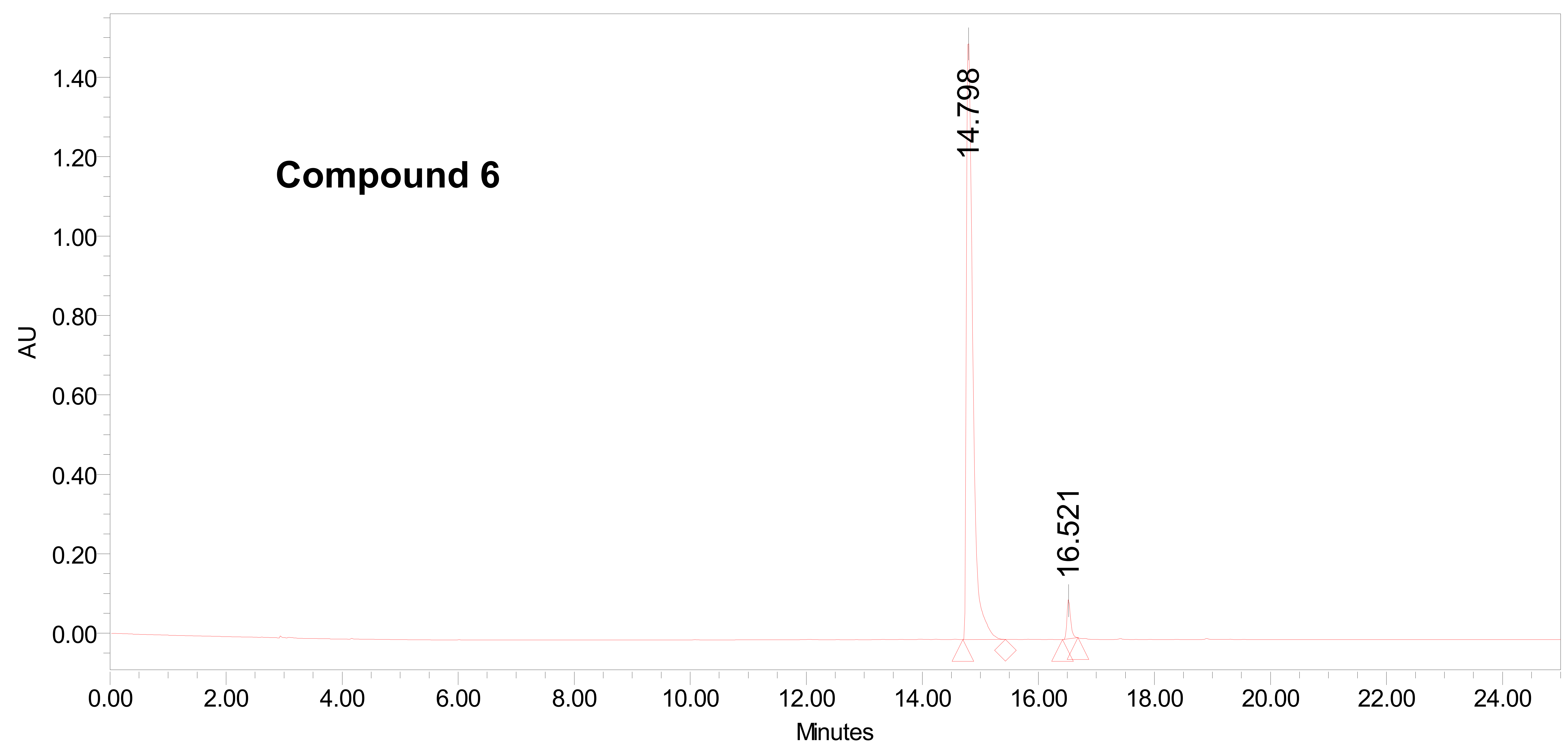

\begin{tabular}{|l|c|r|r|r|}
\multicolumn{5}{c}{ Peak Results } \\
\hline & RT & \multicolumn{1}{|c|}{ Area } & \% Area & Height \\
\hline 1 & 14.798 & 11816741 & 96.42 & 1508849 \\
\hline 2 & 16.521 & 439289 & 3.58 & 96262 \\
\hline
\end{tabular}

Reported by User: walkup Report Method: WalkUpReport Report Method ID 1018

Page: 1 of 1
Project Name: MedChemlMedChem2019-06

Date Printed:

$7 / 26 / 2019$

12:41:42 PM America/New_York 


\begin{tabular}{|llll|}
\hline & \multicolumn{1}{c|}{ S A M P L E I N F O R M A T I O N } \\
\hline \hline Sample Name: & 14014-058 & Acquired By: & walkup \\
Sample Type: & Unknown & Sample Set Name: & thuy20190626 \\
Vial: & 1 & Acq. Method Set: & MethodSetAB5T95B15Min \\
Injection \#: & 1 & Processing Method: & 220W280W15MIN \\
Injection Volume: & $10.00 \mathrm{ul}$ & Channel Name: & W2996 280.0nm-4.0 \\
Run Time: & 30.0 Minutes & Proc. Chnl. Descr.: & W2996 PDA 280.0 nm at 4.0 \\
& & & \\
Date Acquired: & $6 / 26 / 201911: 04: 51$ AM EDT & & \\
Date Processed: & $6 / 26 / 201911: 35: 03$ AM EDT & & \\
\hline
\end{tabular}

Auto-Scaled Chromatogram

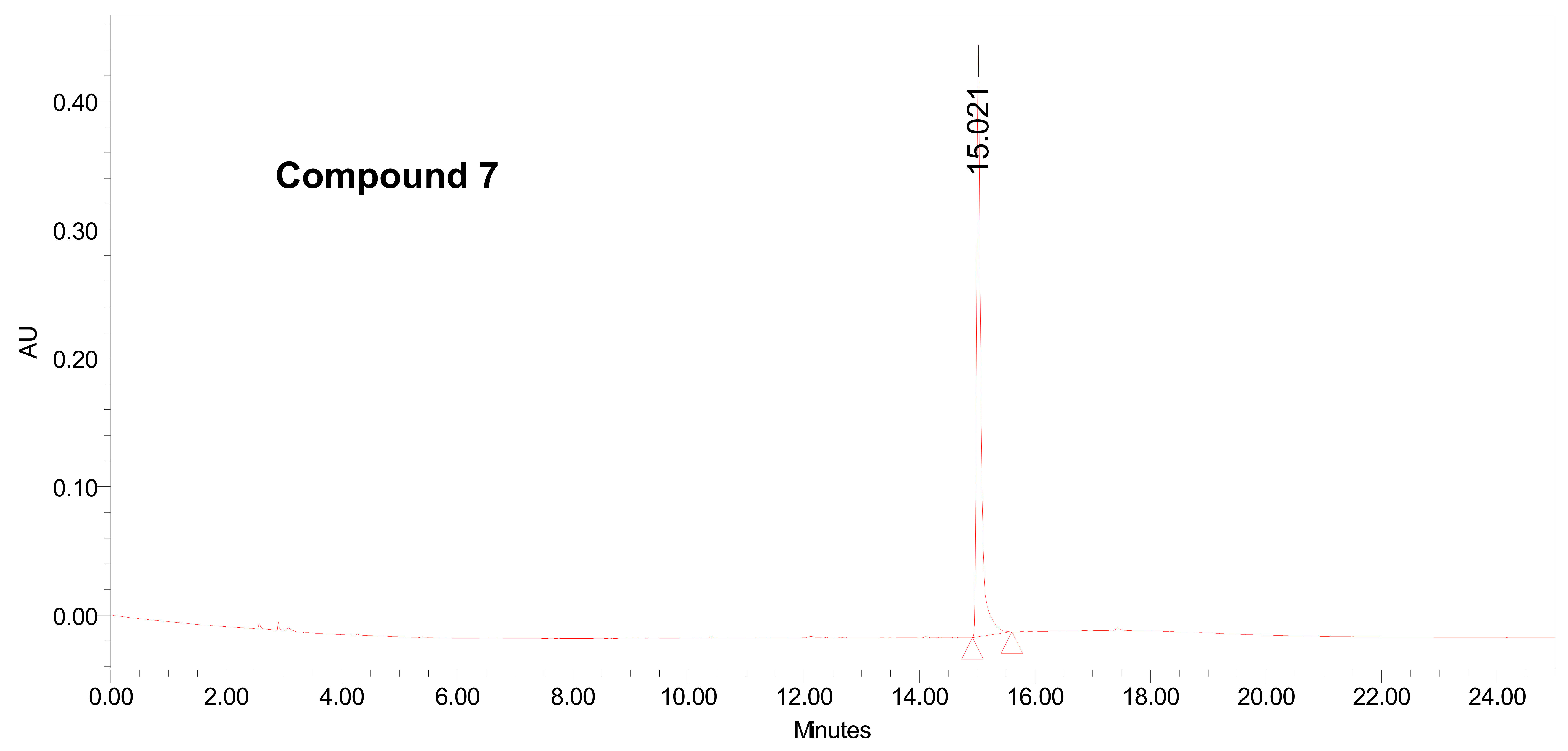

\begin{tabular}{|} 
Peak Results \\
\begin{tabular}{|c|c|c|c|c|}
\hline & RT & Area & $\%$ Area & Height \\
\hline 1 & 15.021 & 2425630 & 100.00 & 451651 \\
\hline
\end{tabular}
\end{tabular}

Reported by User: walkup

Report Method: WalkUpReport

Report Method ID 1018

Page: 1 of 1
Project Name: MedChemlMedChem2019-06

Date Printed:

6/26/2019

11:51:52 AM America/New_York 


\begin{tabular}{|llll|}
\hline & \multicolumn{1}{c|}{ S A M P E } & I N F O R M A T I O N \\
\hline \hline Sample Name: & $14014-059$ & Acquired By: & walkup \\
Sample Type: & Unknown & Sample Set Name: & thuy20190726d \\
Vial: & 90 & Acq. Method Set: & MethodSetAB5T95B15Min \\
Injection \#: & 1 & Processing Method: & 220W280W15MIN \\
Injection Volume: & $10.00 \mathrm{ul}$ & Channel Name: & W2996 280.0nm-4.0 \\
Run Time: & 30.0 Minutes & Proc. Chnl. Descr.: & W2996 PDA 280.0 nm at 4.0 \\
& & & \\
Date Acquired: & $7 / 26 / 2019$ 11:33:47 AM EDT & & \\
Date Processed: & $7 / 26 / 2019$ 12:03:59 PM EDT & & \\
\hline
\end{tabular}

\section{Auto-Scaled Chromatogram}

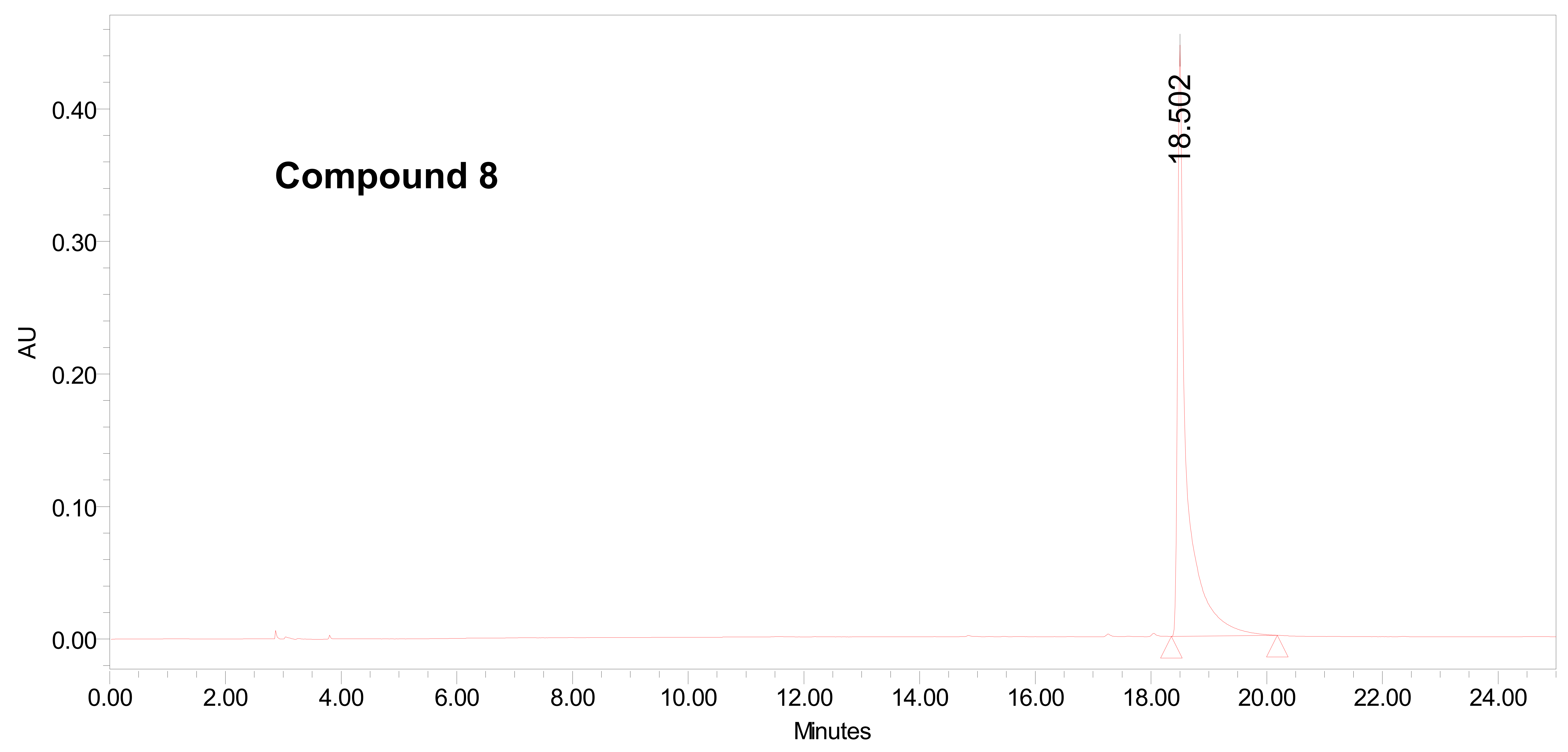

Peak Results
\begin{tabular}{|c|c|c|c|c|}
\hline & RT & Area & $\%$ Area & Height \\
\hline 1 & 18.502 & 4625065 & 100.00 & 442152 \\
\hline
\end{tabular}

Reported by User: walkup Report Method: WalkUpReport Report Method ID 1018

Page: 1 of 1
Project Name: MedChemlMedChem2019-07

Date Printed:

$7 / 26 / 2019$

12:19:07 PM America/New_York 


\begin{tabular}{|llll|}
\hline & \multicolumn{1}{c|}{ S A M P L E } & I N F O R M A T I O N \\
\hline \hline Sample Name: & 14014-099 & Acquired By: & walkup \\
Sample Type: & Unknown & Sample Set Name: & thuy20190625 \\
Vial: & 4 & Acq. Method Set: & MethodSetAB5T95B15Min \\
Injection \#: & 1 & Processing Method: & 220W280W15MIN \\
Injection Volume: & $10.00 \mathrm{ul}$ & Channel Name: & W2996 280.0nm-4.0 \\
Run Time: & 30.0 Minutes & Proc. Chnl. Descr.: & W2996 PDA 280.0 nm at 4.0 \\
& & & \\
Date Acquired: & $6 / 25 / 20196: 18: 11$ PM EDT & & \\
Date Processed: & $6 / 25 / 20196: 48: 24$ PM EDT & & \\
\hline
\end{tabular}

Auto-Scaled Chromatogram

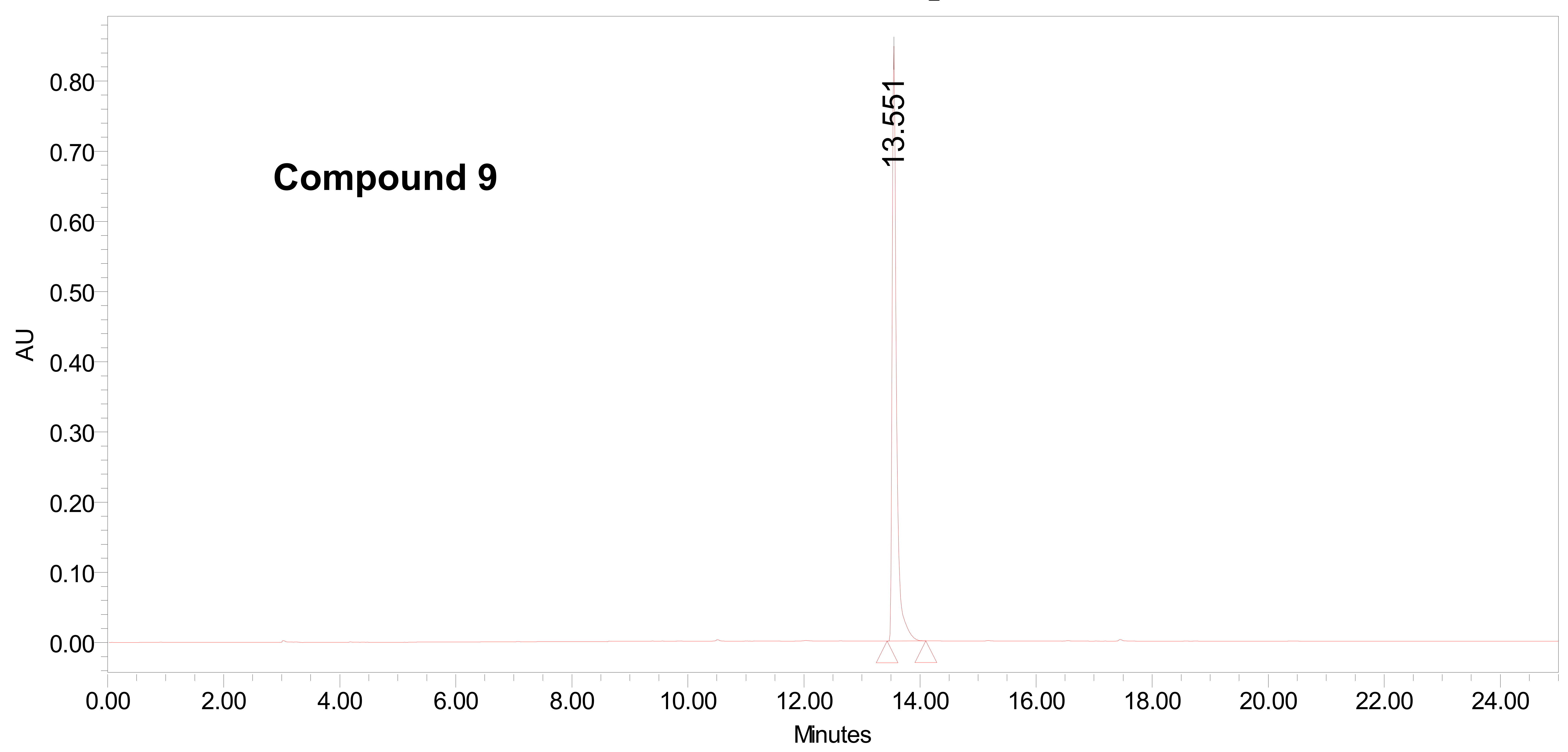

Peak Results
\begin{tabular}{|c|c|c|c|c|}
\hline & RT & Area & $\%$ Area & Height \\
\hline 1 & 13.551 & 4357559 & 100.00 & 836421 \\
\hline
\end{tabular}

Reported by User: walkup

Report Method: WalkUpReport

Report Method ID 1018

Page: 1 of 1
Project Name: MedChemlMedChem2019-06

Date Printed:

6/26/2019

10:50:30 AM America/New_York 


\begin{tabular}{|llll|}
\hline & \multicolumn{1}{c|}{ S A M P L E I N F O R M A T I O N } \\
\hline \hline Sample Name: & 14014-100 & Acquired By: & walkup \\
Sample Type: & Unknown & Sample Set Name: & thuy20190626 \\
Vial: & 2 & Acq. Method Set: & MethodSetAB5T95B15Min \\
Injection \#: & 1 & Processing Method: & 220W280W15MIN \\
Injection Volume: & $50.00 \mathrm{ul}$ & Channel Name: & W2996 280.0nm-4.0 \\
Run Time: & 30.0 Minutes & Proc. Chnl. Descr.: & W2996 PDA 280.0 nm at 4.0 \\
& & & \\
Date Acquired: & $6 / 26 / 2019$ 11:36:04 AM EDT & & \\
Date Processed: & $6 / 26 / 2019$ 12:06:16 PM EDT & \\
\hline
\end{tabular}

\section{Auto-Scaled Chromatogram}

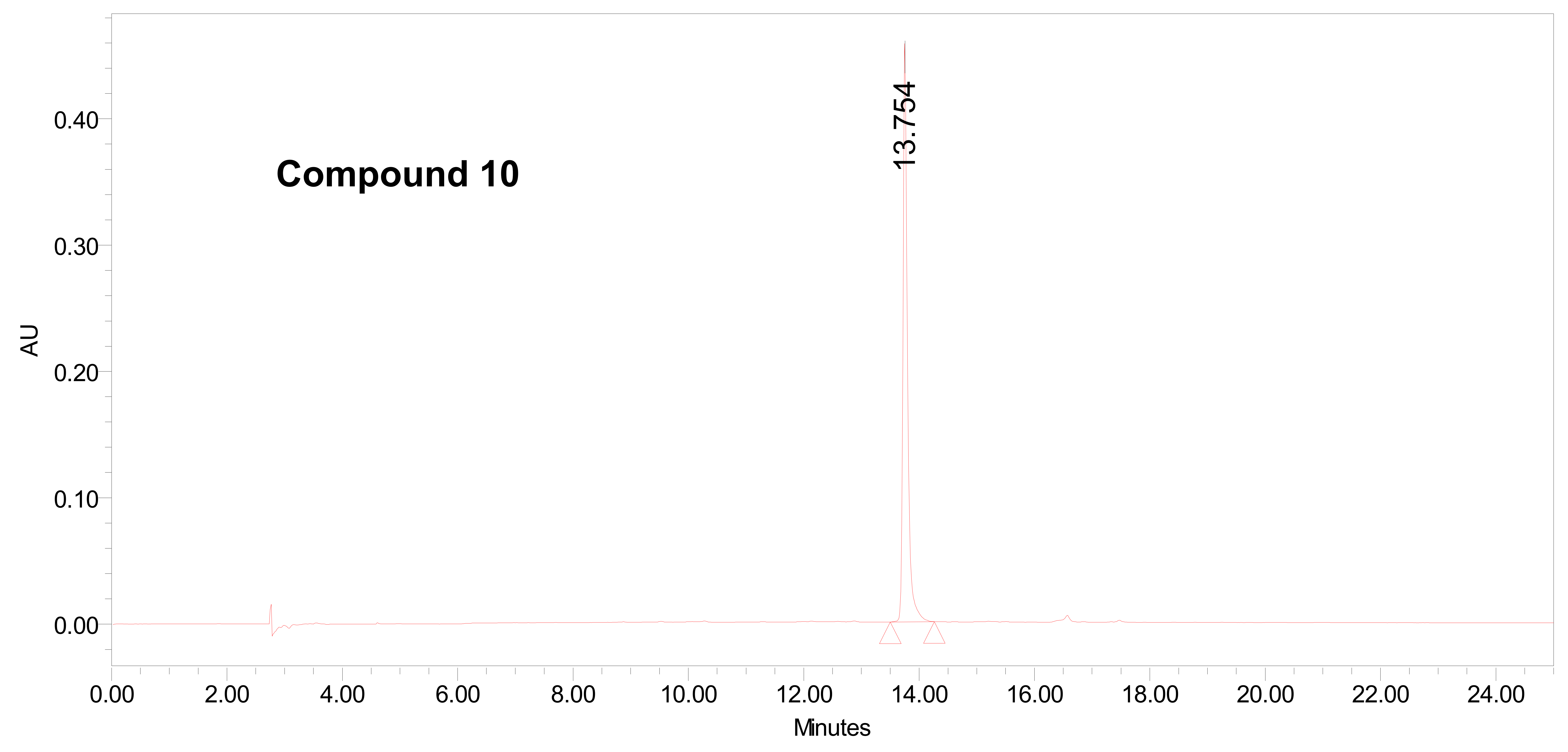

\begin{tabular}{|l|c|c|c|c|}
\multicolumn{5}{c}{ Peak Results } \\
\begin{tabular}{|c|c|c|c|c|}
\hline & RT & Area & $\%$ Area & Height \\
\hline 1 & 13.754 & 2497505 & 100.00 & 451745 \\
\hline
\end{tabular}
\end{tabular}

Reported by User: walkup Report Method: WalkUpReport Report Method ID 1018

Page: 1 of 1
Project Name: MedChemlMedChem2019-06

Date Printed:

6/26/2019

12:26:54 PM America/New_York 


\begin{tabular}{|llll|}
\hline & \multicolumn{1}{c|}{ S A M P E } & I N F O R M A T I O N \\
\hline \hline Sample Name: & 14302-030 & Acquired By: & walkup \\
Sample Type: & Unknown & Sample Set Name: & thuy20180917 \\
Vial: & 5 & Acq. Method Set: & MethodSetAB5T95B15Min \\
Injection \#: & 1 & Processing Method: & 220W280W15MIN \\
Injection Volume: & $10.00 \mathrm{ul}$ & Channel Name: & W2996 280.0nm-4.0 \\
Run Time: & 30.0 Minutes & Proc. Chnl. Descr.: & W2996 PDA 280.0 nm at 4.0 \\
& & & \\
Date Acquired: & $9 / 17 / 2018$ 11:43:17 AM EDT & & \\
Date Processed: & $9 / 17 / 201812: 13: 30$ PM EDT & & \\
\hline
\end{tabular}

\section{Auto-Scaled Chromatogram}

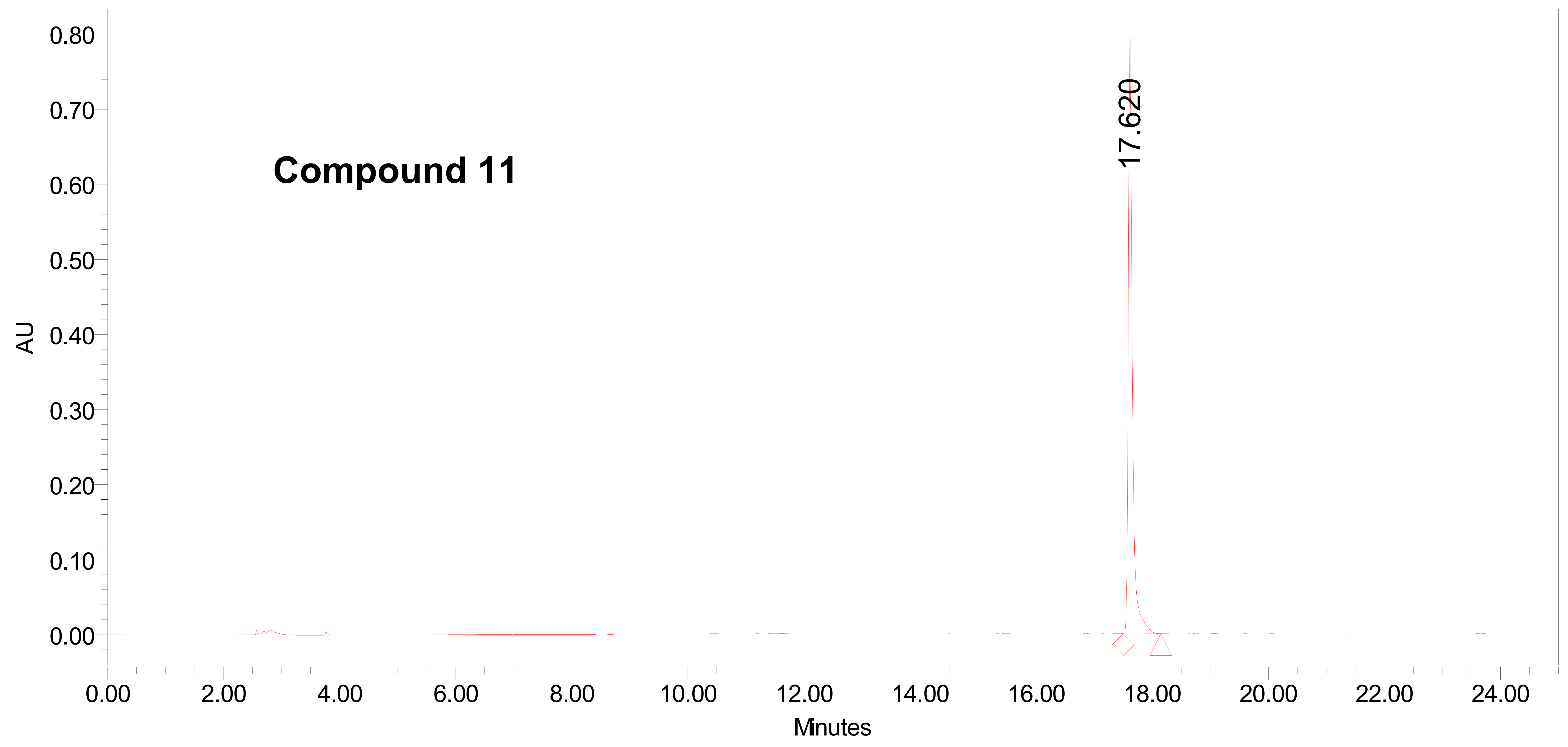

\begin{tabular}{|l|c|c|c|c|}
\multicolumn{5}{c}{ Peak Results } \\
\begin{tabular}{|c|c|c|c|c|}
\hline & RT & Area & $\%$ Area & Height \\
\hline 1 & 17.620 & 3899643 & 100.00 & 768009 \\
\hline
\end{tabular}
\end{tabular}

Reported by User: walkup Report Method: WalkUpReport Report Method ID 1020

Page: 1 of 1
Project Name: MedChemlMedChem2018-09

Date Printed:

9/17/2018

12:34:16 PM America/New_York 


\begin{tabular}{|llll|}
\hline & \multicolumn{2}{c|}{ S A M P LE } & I N F O R M A T I O N \\
\hline \hline Sample Name: & 14125-024 & Acquired By: & walkup \\
Sample Type: & Unknown & Sample Set Name: & thuy20170419 \\
Vial: & 26 & Acq. Method Set: & MethodSetAB5T95B15Min \\
Injection \#: & 1 & Processing Method & 220W280W15MIN \\
Injection Volume: & 10.00 ul & Channel Name: & W2996 280.0nm-4.0 \\
Run Time: & 30.0 Minutes & Proc. Chnl. Descr.: W2996 PDA 280.0 nm at 4.0 \\
& & & \\
Date Acquired: & $4 / 19 / 201711: 22: 18$ AM EDT & & \\
Date Processed: & $4 / 19 / 201711: 52: 31$ AM EDT & & \\
\hline
\end{tabular}

\section{Auto-Scaled Chromatogram}

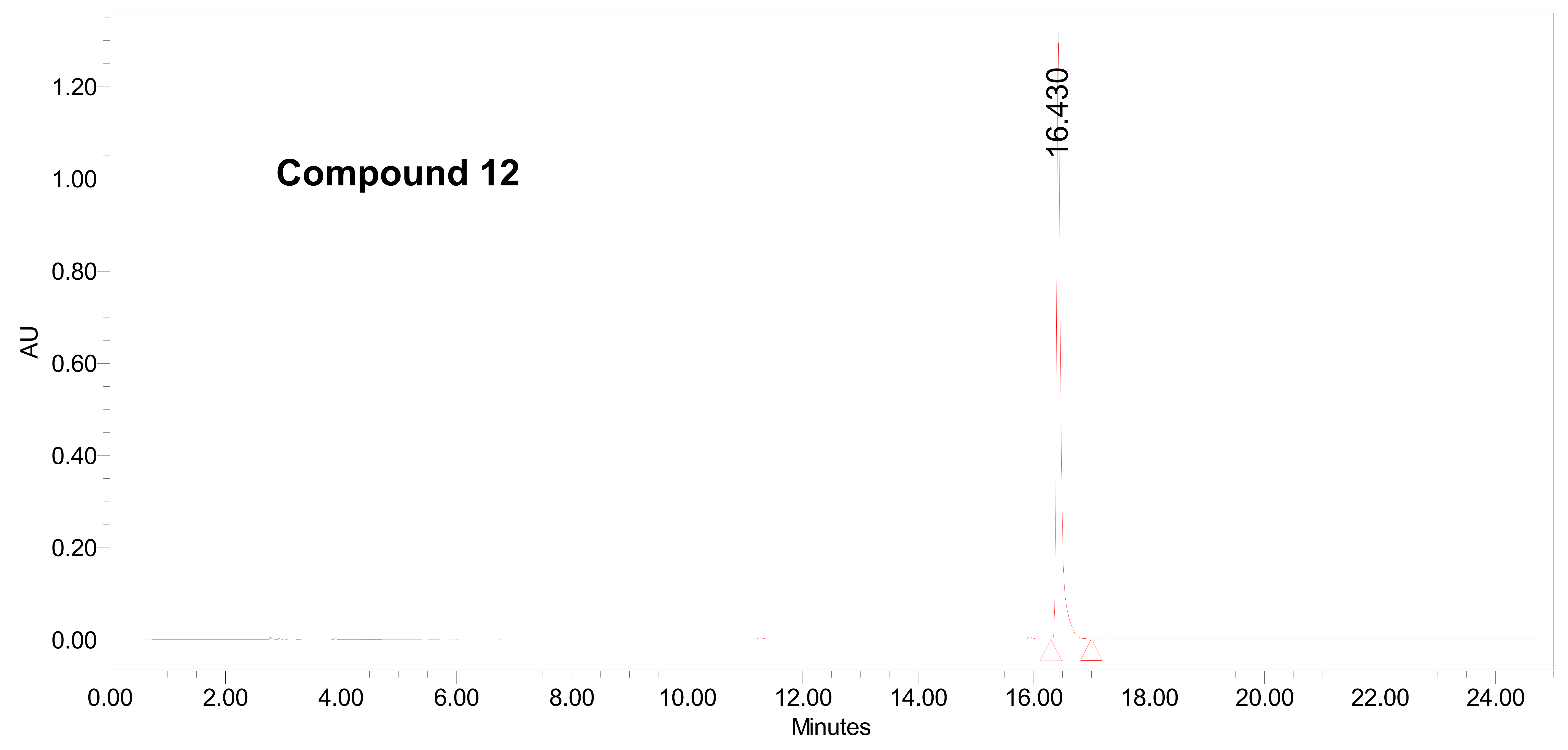

\begin{tabular}{|c|c|c|c|c|}
\hline \multicolumn{5}{|c|}{ Peak Results } \\
\hline & RT & Area & $\%$ Area & Height \\
\hline 1 & 16.430 & 6583947 & 100.00 & 1268535 \\
\hline
\end{tabular}




\begin{tabular}{|llll|}
\hline & \multicolumn{1}{c|}{ S A M P LE } & I N F O R M A T I O N \\
\hline \hline Sample Name: & 14200-158 & Acquired By: & walkup \\
Sample Type: & Unknown & Sample Set Name: & thuy20180321 \\
Vial: & 20 & Acq. Method Set: & MethodSetAB5T95B15Min \\
Injection \#: & 1 & Processing Method & 220W280W15MIN \\
Injection Volume: & $10.00 \mathrm{ul}$ & Channel Name: & W2996 280.0nm-4.0 \\
Run Time: & 30.0 Minutes & Proc. Chnl. Descr.: & W2996 PDA 280.0 nm at 4.0 \\
& & & \\
Date Acquired: & $3 / 21 / 201811: 17: 51$ AM EST & & \\
Date Processed: & $3 / 21 / 201811: 48: 04$ AM EST & & \\
\hline
\end{tabular}

\section{Auto-Scaled Chromatogram}

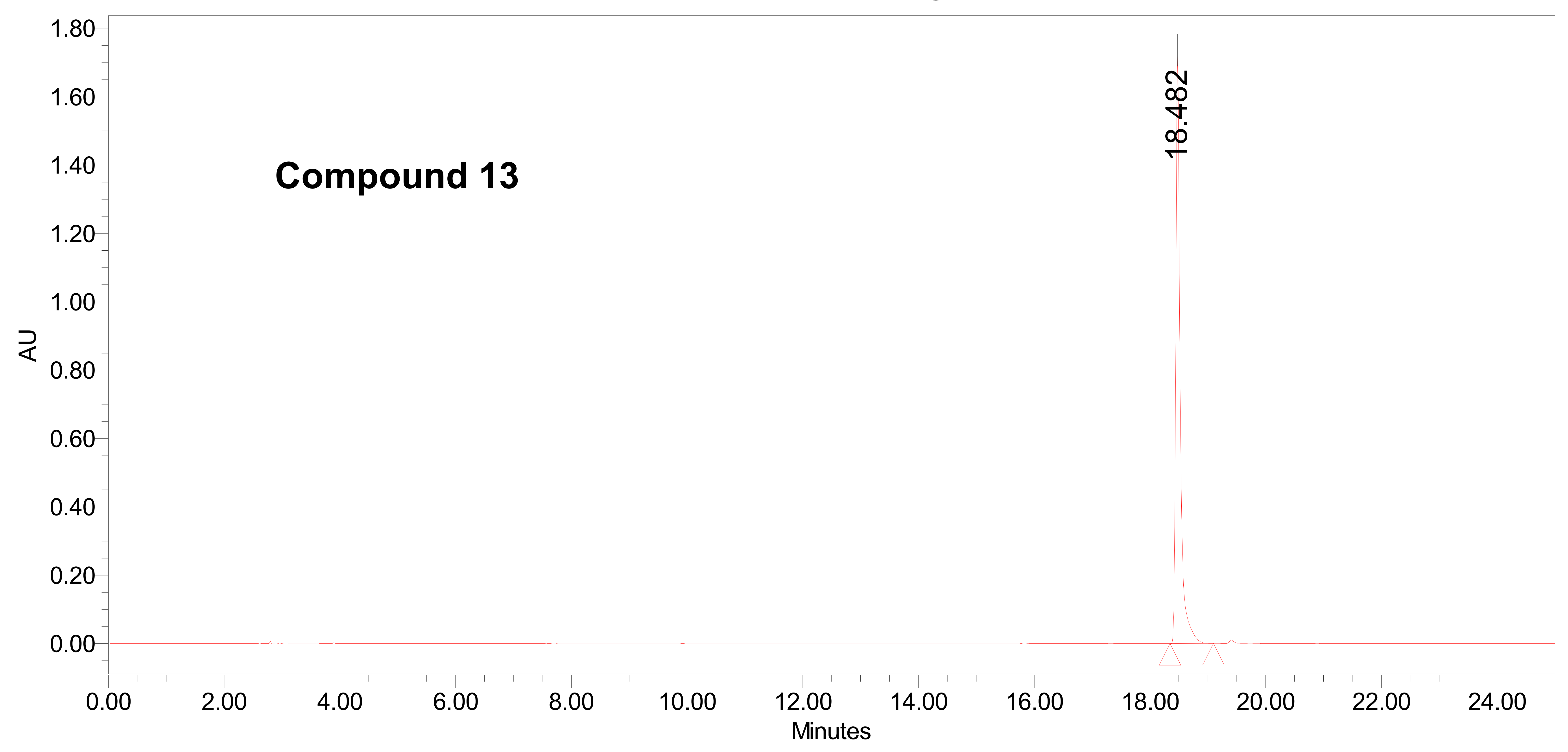

\begin{tabular}{|c|c|c|c|c|}
\multicolumn{5}{c}{ Peak Results } \\
\begin{tabular}{|c|c|c|c|c|}
\hline & RT & Area & $\%$ Area & Height \\
\hline 1 & 18.482 & 9299812 & 100.00 & 1710868 \\
\hline
\end{tabular}
\end{tabular}




\begin{tabular}{|llll|}
\hline & \multicolumn{2}{c|}{ S A M P LE } & I N F O R M A T I O N \\
\hline \hline Sample Name: & 14125-027 & Acquired By: & walkup \\
Sample Type: & Unknown & Sample Set Name: & thuy20170421 \\
Vial: & 1 & Acq. Method Set: & MethodSetAB5T95B15Min \\
Injection \#: & 1 & Processing Method & 220W280W15MIN \\
Injection Volume: & $10.00 \mathrm{ul}$ & Channel Name: & W2996 280.0nm-4.0 \\
Run Time: & 30.0 Minutes & Proc. Chnl. Descr.: & W2996 PDA 280.0 nm at 4.0 \\
& & & \\
Date Acquired: & $4 / 21 / 201710: 24: 57$ AM EDT & & \\
Date Processed: & $4 / 21 / 201710: 55: 08$ AM EDT & & \\
\hline
\end{tabular}

\section{Auto-Scaled Chromatogram}

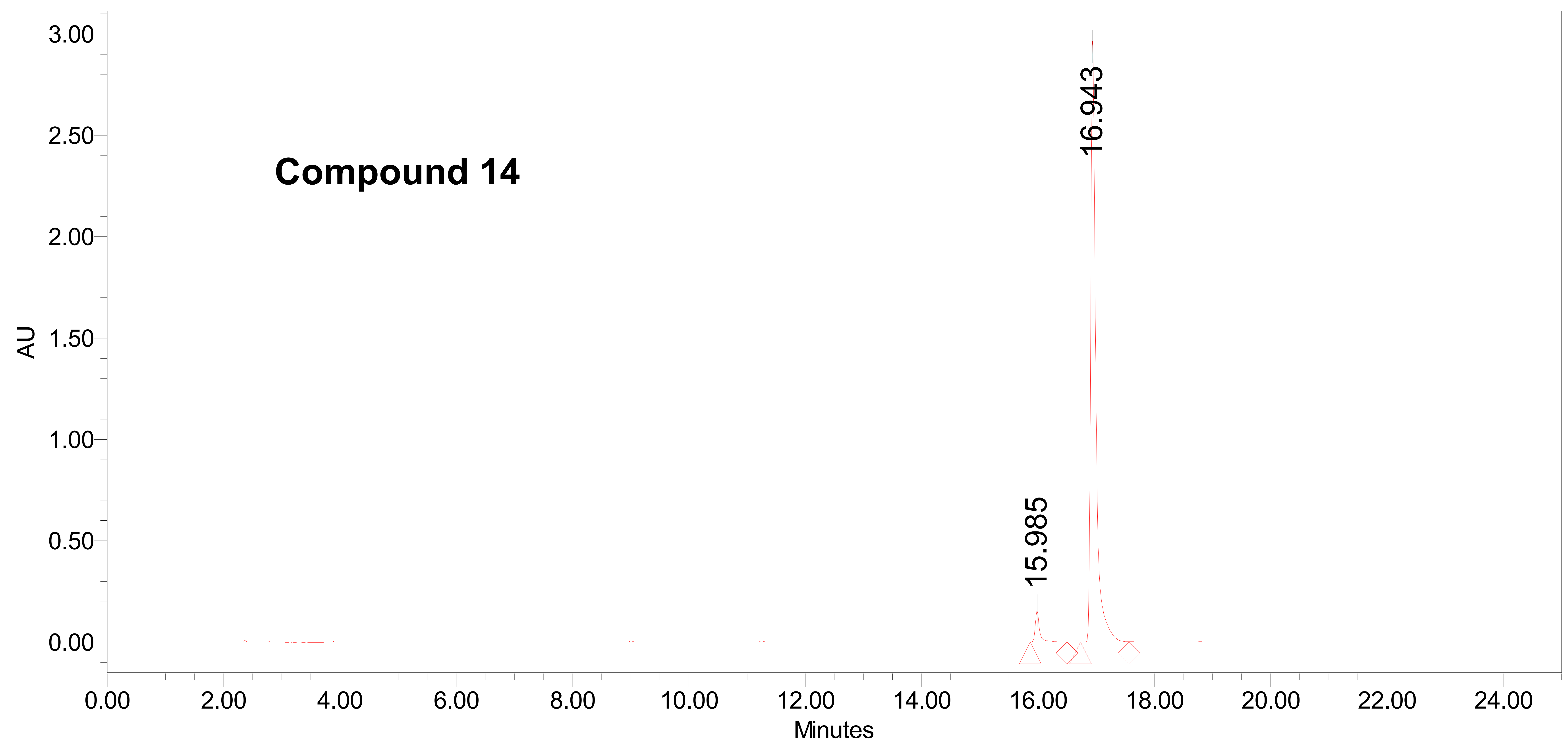

\begin{tabular}{|l|c|c|r|r|}
\multicolumn{5}{c|}{ Peak Results } \\
\hline & RT & Area & $\%$ Area & Height \\
\hline 1 & 15.985 & 778456 & 4.07 & 152750 \\
\hline 2 & 16.943 & 18325034 & 95.93 & 2993312 \\
\hline
\end{tabular}




\begin{tabular}{|llll|}
\hline & \multicolumn{1}{c|}{ S A M P L E I N F O R M A T I O N } \\
\hline \hline Sample Name: & 14014-162 & Acquired By: & walkup \\
Sample Type: & Unknown & Sample Set Name: & thuy20190726c \\
Vial: & 90 & Acq. Method Set: & MethodSetAB5T95B15Min \\
Injection \#: & 1 & Processing Method: & 220W280W15MIN \\
Injection Volume: & $10.00 \mathrm{ul}$ & Channel Name: & W2996 220.0nm-2.0 \\
Run Time: & 30.0 Minutes & Proc. Chnl. Descr.: & W2996 PDA 220.0 nm at 2.0 \\
& & & \\
Date Acquired: & $7 / 26 / 201911: 02: 34$ AM EDT & & \\
Date Processed: & $7 / 26 / 201911: 35: 13$ AM EDT & & \\
\hline
\end{tabular}

Auto-Scaled Chromatogram

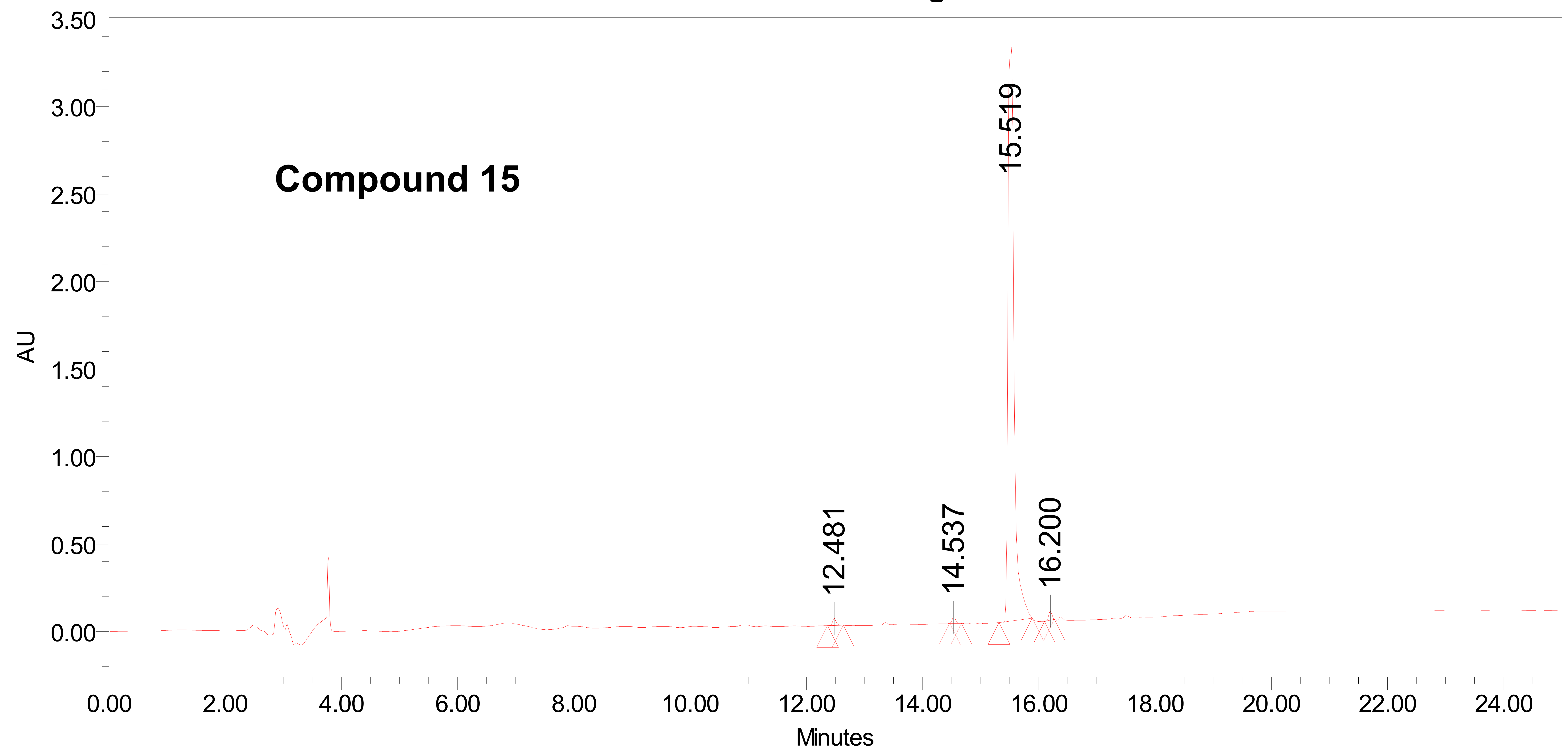

\begin{tabular}{|l|c|r|r|r|}
\multicolumn{5}{|c}{ Peak Results } \\
\hline & RT & Area & $\%$ Area & \multicolumn{1}{c|}{ Height } \\
\hline 1 & 12.481 & 176517 & 0.73 & 39920 \\
\hline 2 & 14.537 & 162119 & 0.67 & 35475 \\
\hline 3 & 15.519 & 23750999 & 97.80 & 3367139 \\
\hline 4 & 16.200 & 194668 & 0.80 & 49632 \\
\hline
\end{tabular}

Reported by User: walkup

Report Method: WalkUpReport

Report Method ID 1018

Page: 1 of 1
Project Name: MedChemlMedChem2019-07

Date Printed:

$7 / 26 / 2019$

11:36:14 AM America/New_York 


\begin{tabular}{|llll|}
\hline & \multicolumn{1}{c|}{ S A M P E } & I N F O R M A T I O N \\
\hline \hline Sample Name: & $14200-087$ & Acquired By: & walkup \\
Sample Type: & Unknown & Sample Set Name: & thuy20171121a \\
Vial: & 1 & Acq. Method Set: & MethodSetAB5T95B15Min \\
Injection \#: & 1 & Processing Method: & 220W280W15MIN \\
Injection Volume: & $20.00 \mathrm{ul}$ & Channel Name: & W2996 280.0nm-4.0 \\
Run Time: & 30.0 Minutes & Proc. Chnl. Descr.: & W2996 PDA 280.0 nm at 4.0 \\
& & & \\
Date Acquired: & $11 / 21 / 201712: 33: 19$ PM EST & & \\
Date Processed: & $7 / 26 / 20191: 20: 10$ PM EDT & & \\
\hline
\end{tabular}

\section{Auto-Scaled Chromatogram}

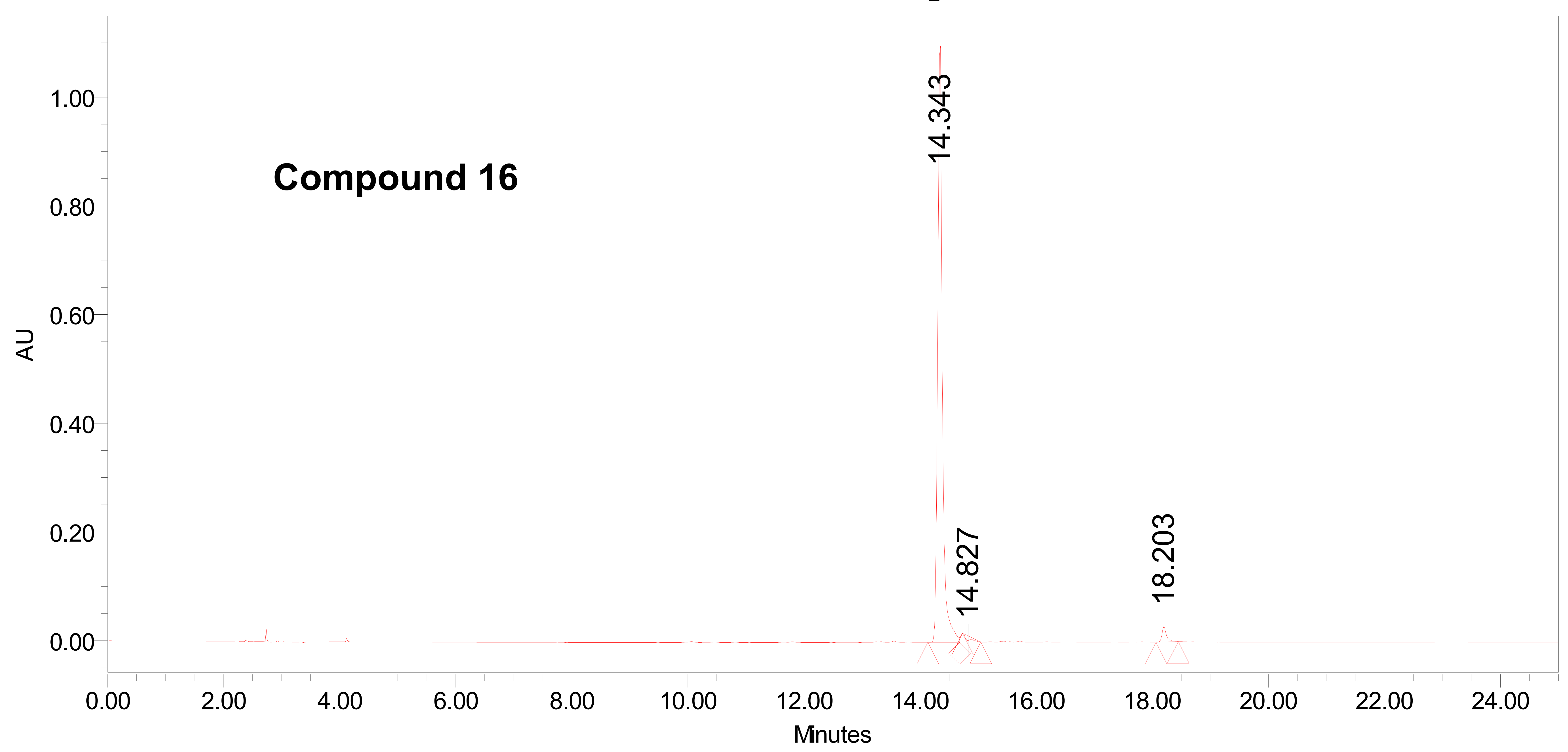

\begin{tabular}{|l|c|r|r|r|}
\multicolumn{5}{|c|}{ Peak Results } \\
\hline & RT & Area & $\%$ Area & Height \\
\hline 1 & 14.343 & 6243027 & 96.64 & 1089955 \\
\hline 2 & 14.827 & 69255 & 1.07 & -8700 \\
\hline 3 & 18.203 & 148083 & 2.29 & 27988 \\
\hline
\end{tabular}

Reported by User: walkup Report Method: WalkUpReport Report Method ID 1022

Page: 1 of 1
Project Name: $\quad$ MedChemlMedChem2017-11

Date Printed:

$7 / 26 / 2019$

1:20:58 PM America/New_York 


\begin{tabular}{|llll|}
\hline & \multicolumn{1}{c|}{ S A M P L E I N F O R M A T I O N } \\
\hline \hline Sample Name: & 14200-053 & Acquired By: & walkup \\
Sample Type: & Unknown & Sample Set Name: & thuy20190726e \\
Vial: & 90 & Acq. Method Set: & MethodSetAB5T95B15Min \\
Injection \#: & 1 & Processing Method: & 220W280W15MIN \\
Injection Volume: & $10.00 \mathrm{ul}$ & Channel Name: & W2996 280.0nm-4.0 \\
Run Time: & 30.0 Minutes & Proc. Chnl. Descr.: & W2996 PDA 280.0 nm at 4.0 \\
& & & \\
Date Acquired: & $7 / 26 / 2019$ 12:04:56 PM EDT & & \\
Date Processed: & $7 / 26 / 2019$ 12:37:25 PM EDT & & \\
\hline
\end{tabular}

Auto-Scaled Chromatogram

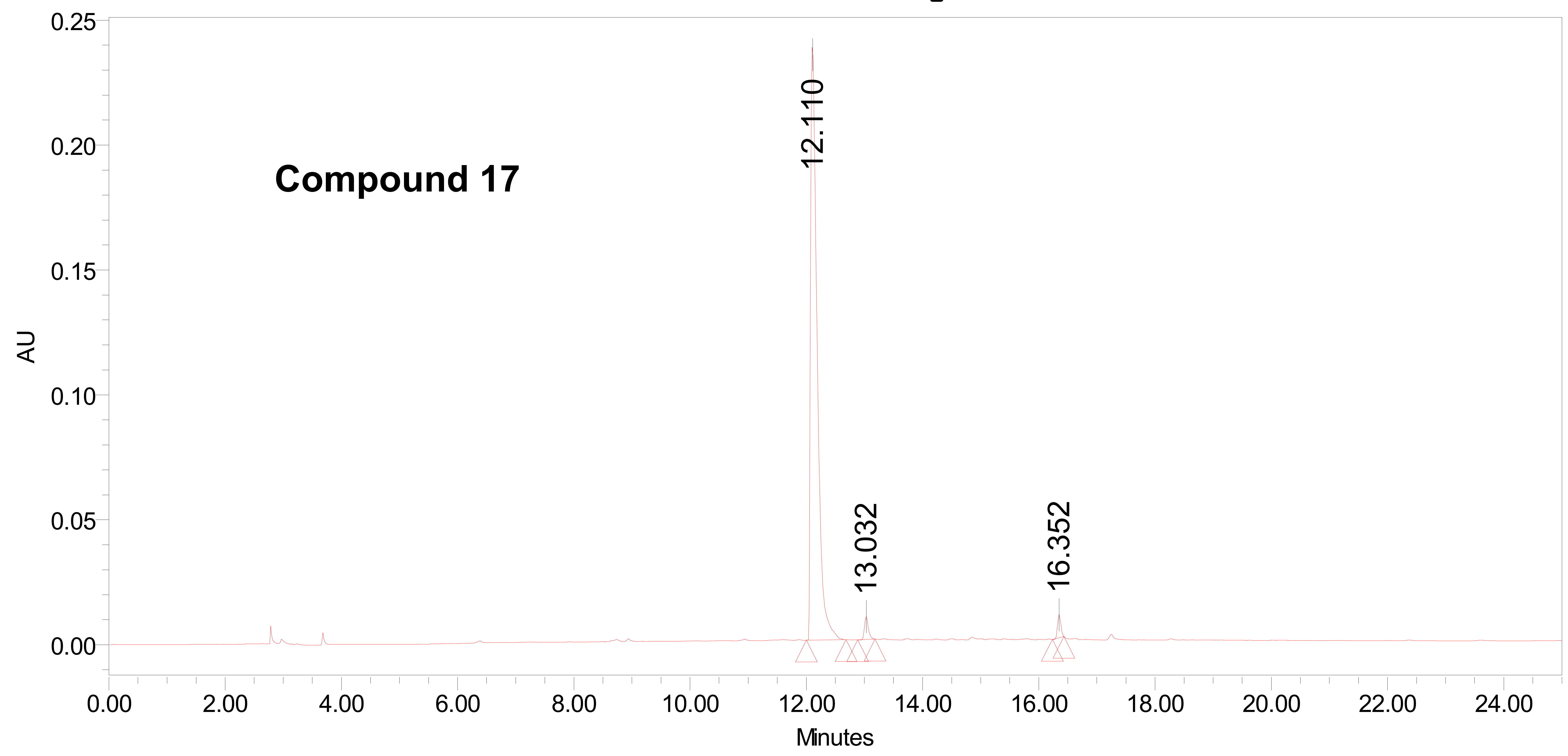

\begin{tabular}{|l|c|r|r|r|}
\multicolumn{9}{|c|}{ Peak Results } \\
\hline & RT & \multicolumn{1}{|c|}{ Area } & $\%$ Area & Height \\
\hline 1 & 12.110 & 1889185 & 95.98 & 245908 \\
\hline 2 & 13.032 & 41727 & 2.12 & 8955 \\
\hline 3 & 16.352 & 37489 & 1.90 & 9068 \\
\hline
\end{tabular}

Reported by User: walkup

Report Method: WalkUpReport

Report Method ID 1018

Page: 1 of 1
Project Name: MedChemlMedChem2019-07

Date Printed:

$7 / 26 / 2019$

12:37:41 PM America/New_York 


\begin{tabular}{|llll|}
\hline & \multicolumn{1}{c|}{ S A M P E } & I N F O R M A T I O N \\
\hline \hline Sample Name: & $14125-012$ & Acquired By: & walkup \\
Sample Type: & Unknown & Sample Set Name: & thuy20170417 \\
Vial: & 1 & Acq. Method Set: & MethodSetAB5T95B15Min \\
Injection \#: & 1 & Processing Method: & 220W280W15MIN \\
Injection Volume: & $10.00 \mathrm{ul}$ & Channel Name: & W2996 280.0nm-4.0 \\
Run Time: & 30.0 Minutes & Proc. Chnl. Descr.: & W2996 PDA 280.0 nm at 4.0 \\
& & & \\
Date Acquired: & $4 / 17 / 2017$ 10:34:17 AM EDT & & \\
Date Processed: & $7 / 26 / 2019$ 12:49:25 PM EDT & & \\
\hline
\end{tabular}

\section{Auto-Scaled Chromatogram}

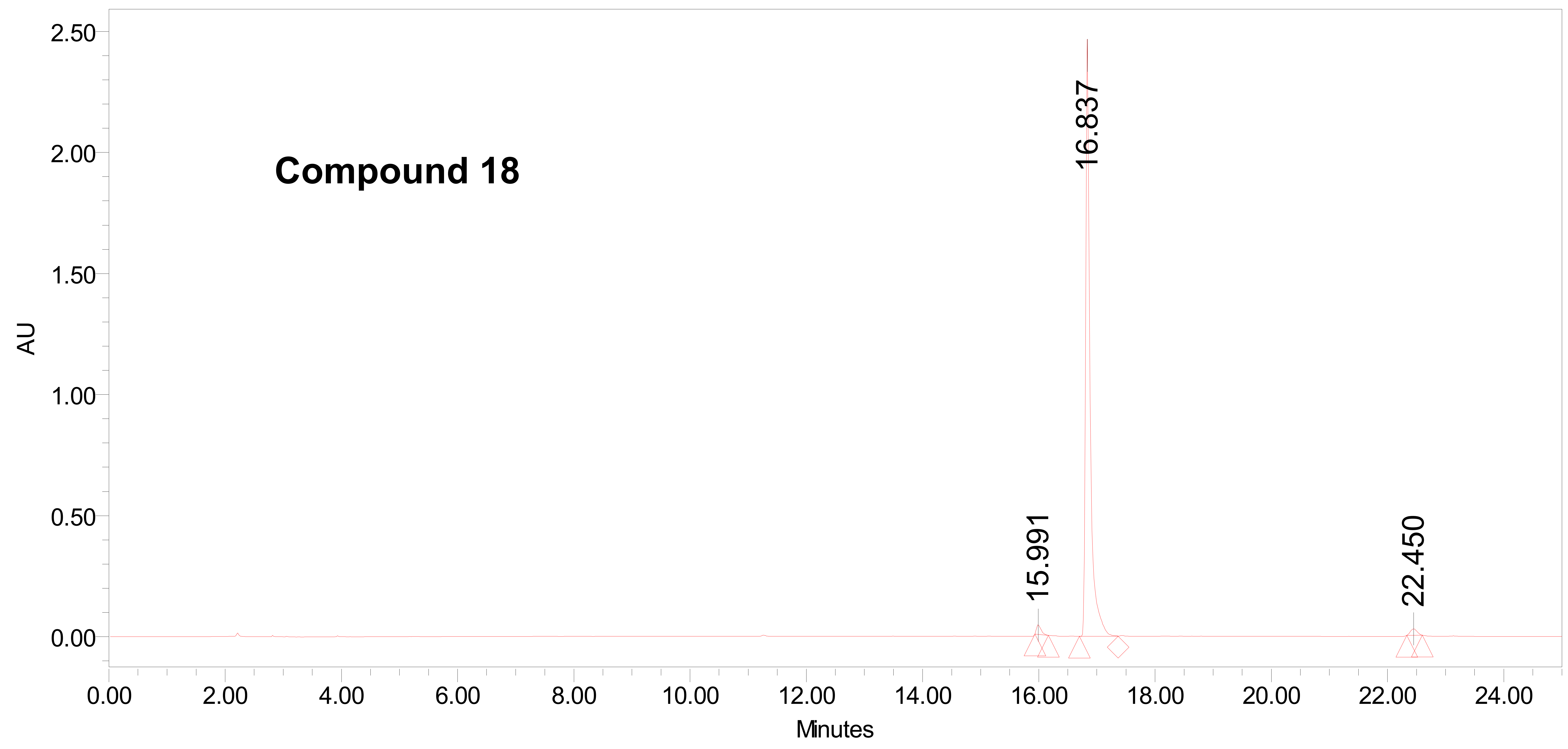

\begin{tabular}{|l|c|r|r|r|}
\multicolumn{5}{|c|}{ Peak Results } \\
\hline & RT & \multicolumn{1}{|c|}{ Area } & $\%$ Area & \multicolumn{1}{|c|}{ Height } \\
\hline 1 & 15.991 & 214506 & 1.57 & 39115 \\
\hline 2 & 16.837 & 13232258 & 96.87 & 2399520 \\
\hline 3 & 22.450 & 212602 & 1.56 & 26689 \\
\hline
\end{tabular}

Reported by User: walkup Report Method: WalkUpReport Report Method ID 1098

Page: 1 of 1
Project Name: MedChemlMedChem2017-04

Date Printed:

$7 / 26 / 2019$

12:49:36 PM America/New_York 


\begin{tabular}{|llll|}
\hline & \multicolumn{1}{c|}{ S A M P E } & I N F O R M A T I O N \\
\hline \hline Sample Name: & $14125-079$ & Acquired By: & walkup \\
Sample Type: & Unknown & Sample Set Name: & thuy20170519a \\
Vial: & 30 & Acq. Method Set: & MethodSetAB5T95B15Min \\
Injection \#: & 1 & Processing Method: & 220W280W15MIN \\
Injection Volume: & $10.00 \mathrm{ul}$ & Channel Name: & W2996 280.0nm-4.0 \\
Run Time: & 30.0 Minutes & Proc. Chnl. Descr.: & W2996 PDA 280.0 nm at 4.0 \\
& & & \\
Date Acquired: & $5 / 19 / 20172: 23: 55$ PM EDT & & \\
Date Processed: & $7 / 26 / 2019$ 1:39:39 PM EDT & & \\
\hline
\end{tabular}

\section{Auto-Scaled Chromatogram}

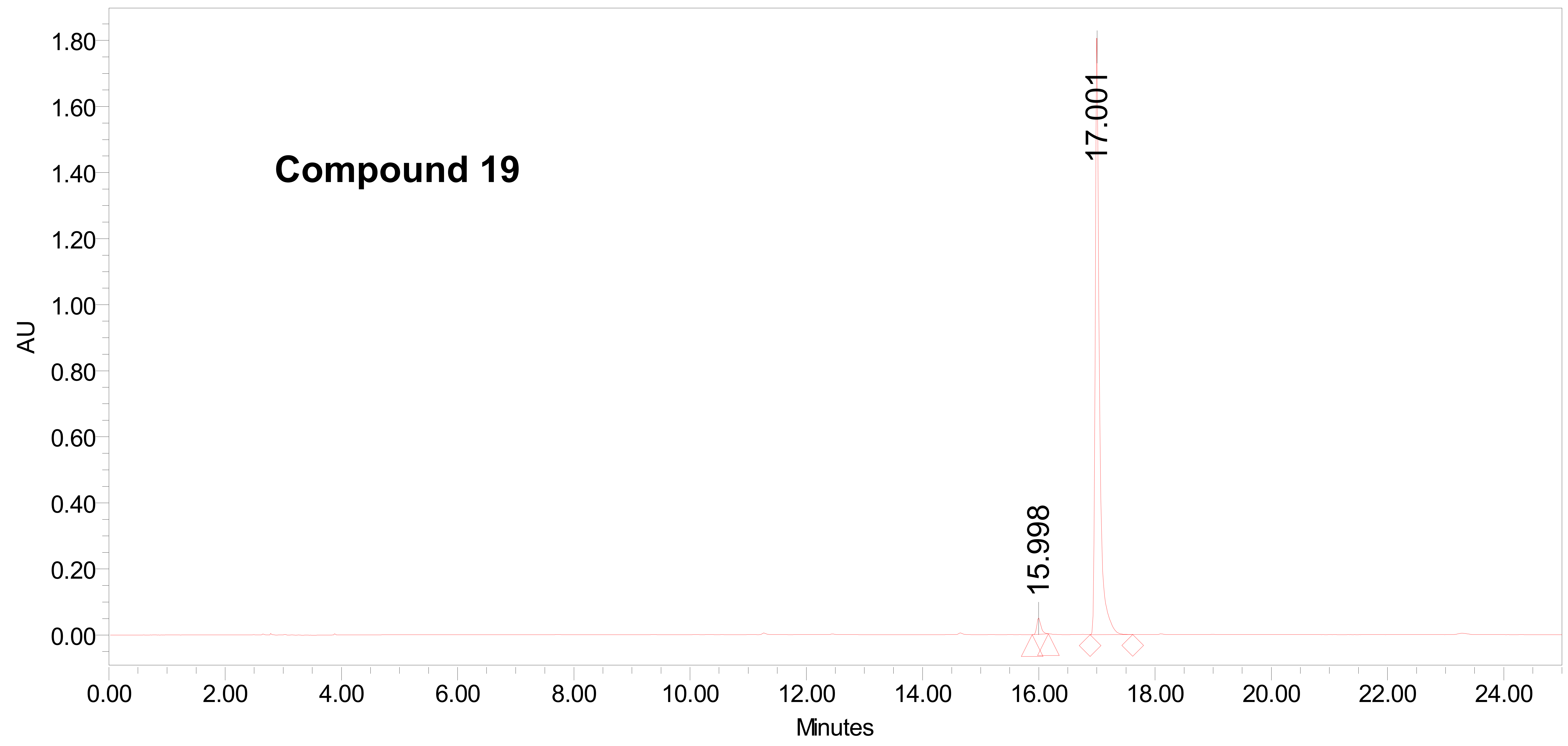

\begin{tabular}{|l|c|c|r|r|}
\multicolumn{9}{c|}{ Peak Results } \\
\hline & RT & Area & $\%$ Area & Height \\
\hline 1 & 15.998 & 245344 & 2.58 & 47902 \\
\hline 2 & 17.001 & 9267511 & 97.42 & 1751737 \\
\hline
\end{tabular}

Reported by User: walkup Report Method: WalkUpReport Report Method ID 1022

Page: 1 of 1
Project Name: MedChemlMedChem2017-05

Date Printed:

$7 / 26 / 2019$

1:39:55 PM America/New_York 


\begin{tabular}{|llll|}
\hline & \multicolumn{1}{c|}{ S A M P E } & I N F O R M A T I O N \\
\hline \hline Sample Name: & $14125-013$ & Acquired By: & walkup \\
Sample Type: & Unknown & Sample Set Name: & thuy20170417 \\
Vial: & 2 & Acq. Method Set: & MethodSetAB5T95B15Min \\
Injection \#: & 1 & Processing Method: & 220W280W15MIN \\
Injection Volume: & $10.00 \mathrm{ul}$ & Channel Name: & W2996 280.0nm-4.0 \\
Run Time: & 30.0 Minutes & Proc. Chnl. Descr.: & W2996 PDA 280.0 nm at 4.0 \\
& & & \\
Date Acquired: & $4 / 17 / 2017$ 11:05:11 AM EDT & & \\
Date Processed: & $7 / 26 / 2019$ 12:50:24 PM EDT & & \\
\hline
\end{tabular}

\section{Auto-Scaled Chromatogram}

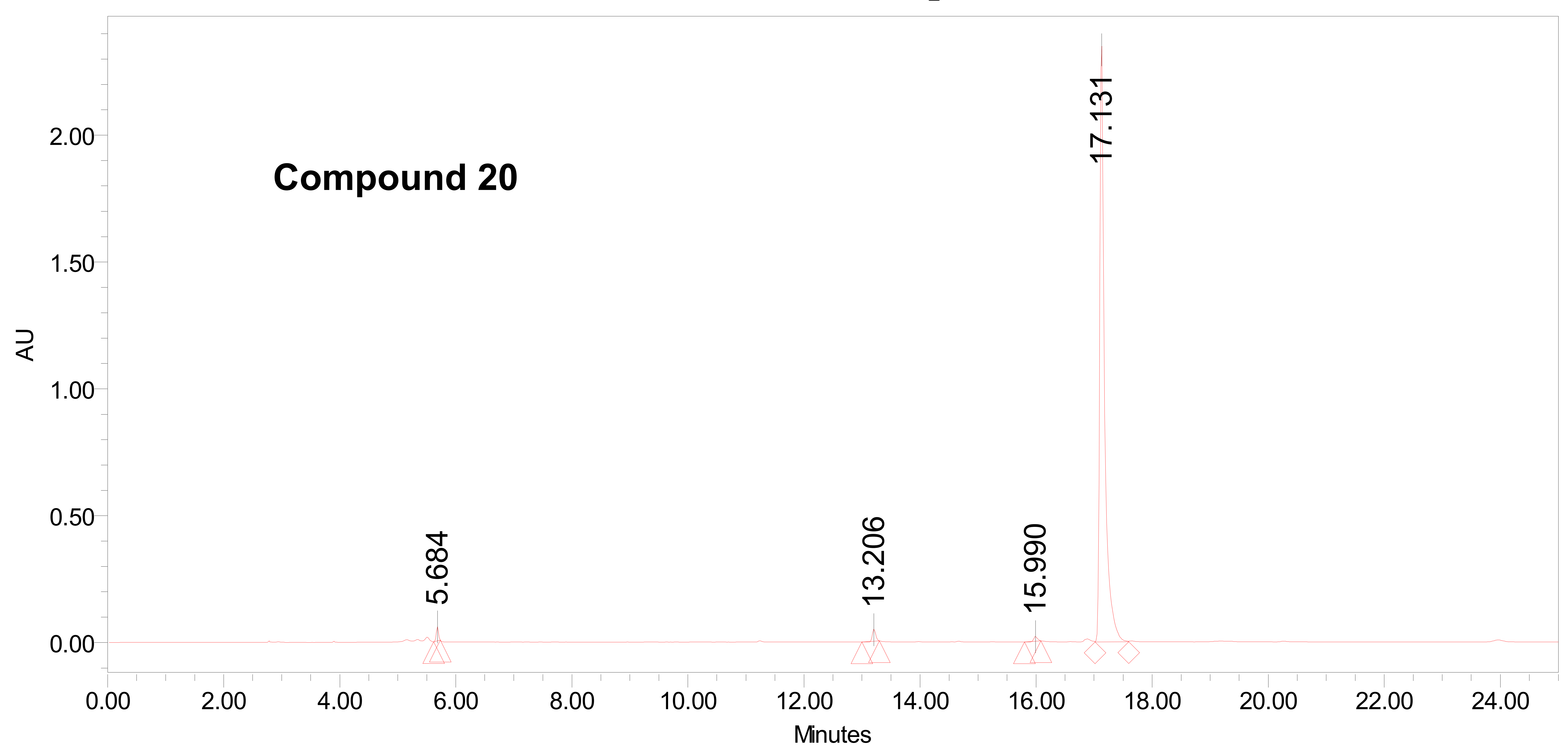

\begin{tabular}{|r|c|r|r|r|}
\multicolumn{5}{|c|}{ Peak Results } \\
\hline & RT & Area & $\%$ Area & Height \\
\hline 1 & 5.684 & 146981 & 1.03 & 49689 \\
\hline 2 & 13.206 & 191252 & 1.34 & 45559 \\
\hline 3 & 15.990 & 87692 & 0.61 & 18130 \\
\hline 4 & 17.131 & 13837824 & 97.01 & 2315646 \\
\hline
\end{tabular}

Reported by User: walkup Report Method: WalkUpReport Report Method ID 1098

Page: 1 of 1
Project Name: MedChemlMedChem2017-04

Date Printed:

$7 / 26 / 2019$

12:50:36 PM America/New_York 


\begin{tabular}{|llll|}
\hline & \multicolumn{1}{c|}{ S A M P E } & I N F O R M A T I O N \\
\hline \hline Sample Name: & $14125-034$ & Acquired By: & walkup \\
Sample Type: & Unknown & Sample Set Name: & thuy20170425 \\
Vial: & 50 & Acq. Method Set: & MethodSetAB5T95B15Min \\
Injection \#: & 1 & Processing Method: & 220W280W15MIN \\
Injection Volume: & $10.00 \mathrm{ul}$ & Channel Name: & W2996 280.0nm-4.0 \\
Run Time: & 30.0 Minutes & Proc. Chnl. Descr.: & W2996 PDA 280.0 nm at 4.0 \\
& & & \\
Date Acquired: & $4 / 26 / 2017$ 11:18:37 AM EDT & & \\
Date Processed: & $7 / 26 / 2019$ 12:53:37 PM EDT & & \\
\hline
\end{tabular}

\section{Auto-Scaled Chromatogram}

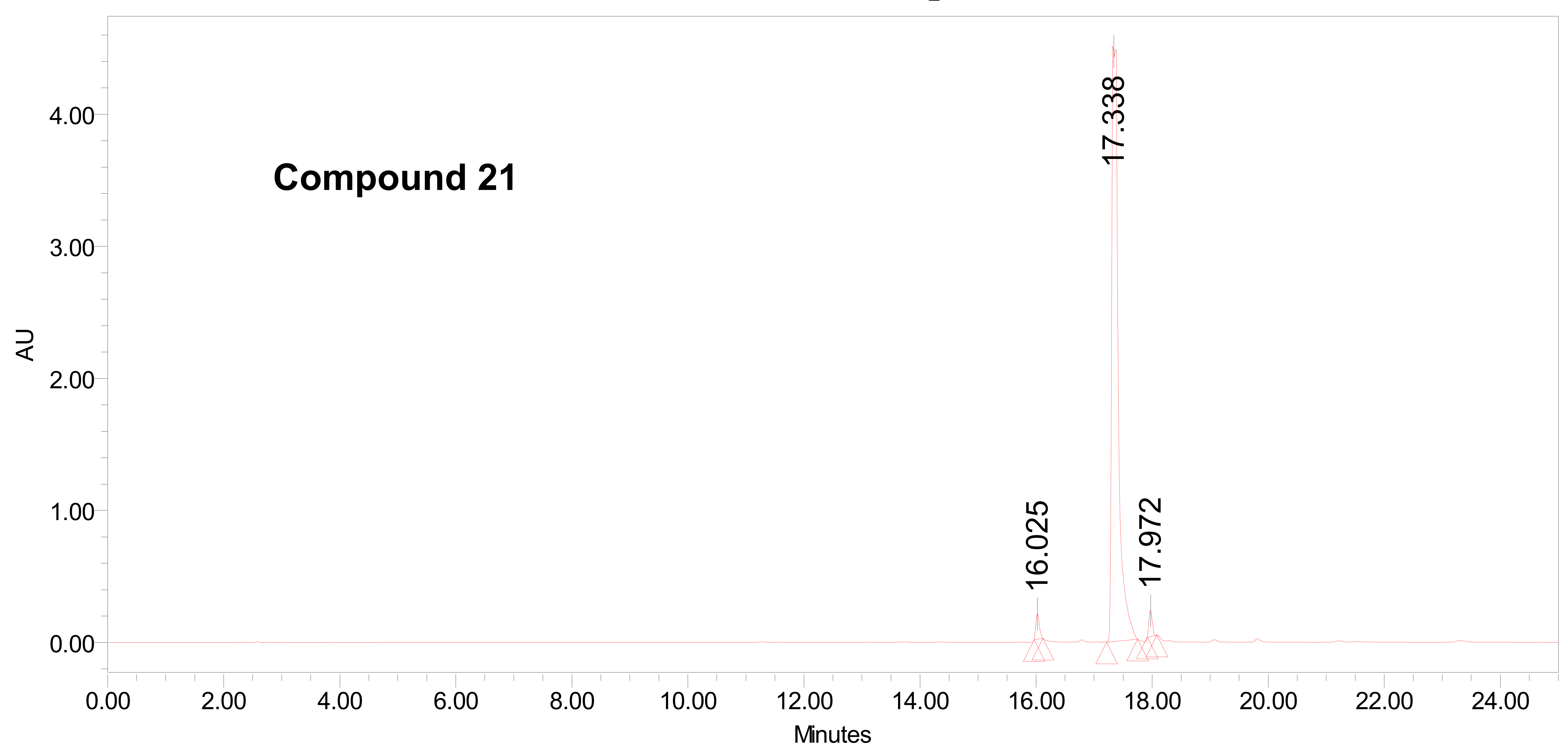

\begin{tabular}{|r|c|r|r|r|}
\multicolumn{5}{|c|}{ Peak Results } \\
\hline & RT & Area & \% Area & Height \\
\hline 1 & 16.025 & 821596 & 2.18 & 190950 \\
\hline 2 & 17.338 & 36088487 & 95.71 & 4641492 \\
\hline 3 & 17.972 & 795541 & 2.11 & 196651 \\
\hline
\end{tabular}

Reported by User: walkup Report Method: WalkUpReport Report Method ID 1098

Page: 1 of 1
Project Name: MedChemlMedChem2017-04

Date Printed:

$7 / 26 / 2019$

12:53:49 PM America/New_York 


\begin{tabular}{|llll|}
\hline & \multicolumn{2}{c|}{ S A M P LE } & I N F O R M A T I O N \\
\hline \hline Sample Name: & 14125-038 & Acquired By: & walkup \\
Sample Type: & Unknown & Sample Set Name: & thuy20170428 \\
Vial: & 110 & Acq. Method Set: & MethodSetAB5T95B15Min \\
Injection \#: & 1 & Processing Method & 220W280W15MIN \\
Injection Volume: & $10.00 \mathrm{ul}$ & Channel Name: & W2996 280.0nm-4.0 \\
Run Time: & 30.0 Minutes & Proc. Chnl. Descr.: & W2996 PDA 280.0 nm at 4.0 \\
& & & \\
Date Acquired: & $4 / 28 / 2017$ 10:00:11 AM EDT & & \\
Date Processed: & $4 / 28 / 201710: 30: 23$ AM EDT & & \\
\hline
\end{tabular}

\section{Auto-Scaled Chromatogram}

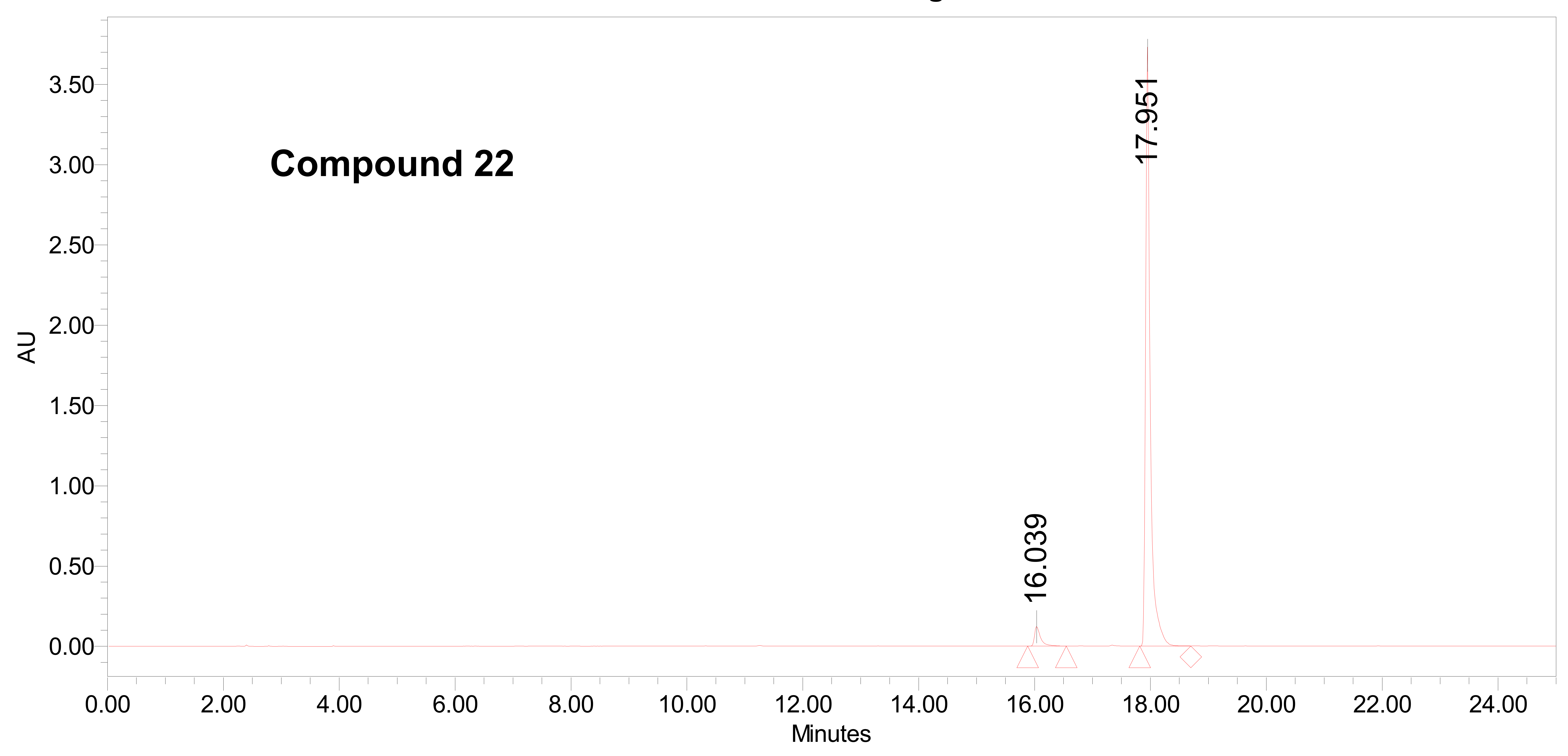

\begin{tabular}{|l|c|r|r|r|}
\multicolumn{5}{|c}{ Peak Results } \\
\begin{tabular}{|c|c|r|r|r|}
\hline & RT & \multicolumn{1}{|c|}{ Area } & $\%$ Area & Height \\
\hline 1 & 16.039 & 902178 & 4.23 & 122776 \\
\hline 2 & 17.951 & 20427822 & 95.77 & 3606426 \\
\hline
\end{tabular}
\end{tabular}

Reported by User: walkup Report Method: WalkUpReport Report Method IL 1098

Page: 1 of 1
Project Name: MedChemlMedChem2017-04

Date Printed: $4 / 28 / 2017$ 10:55:56 AM America/New_York 


\begin{tabular}{|llll|}
\hline & \multicolumn{1}{c|}{ S A M P E } & I N F O R M A T I O N \\
\hline \hline Sample Name: & $14125-039$ & Acquired By: & walkup \\
Sample Type: & Unknown & Sample Set Name: & thuy20170428 \\
Vial: & 111 & Acq. Method Set: & MethodSetAB5T95B15Min \\
Injection \#: & 1 & Processing Method: & 220W280W15MIN \\
Injection Volume: & $10.00 \mathrm{ul}$ & Channel Name: & W2996 280.0nm-4.0 \\
Run Time: & 30.0 Minutes & Proc. Chnl. Descr.: & W2996 PDA 280.0 nm at 4.0 \\
& & & \\
Date Acquired: & $4 / 28 / 2017$ 10:31:11 AM EDT & & \\
Date Processed: & $7 / 26 / 20191: 42: 45$ PM EDT & & \\
\hline
\end{tabular}

\section{Auto-Scaled Chromatogram}

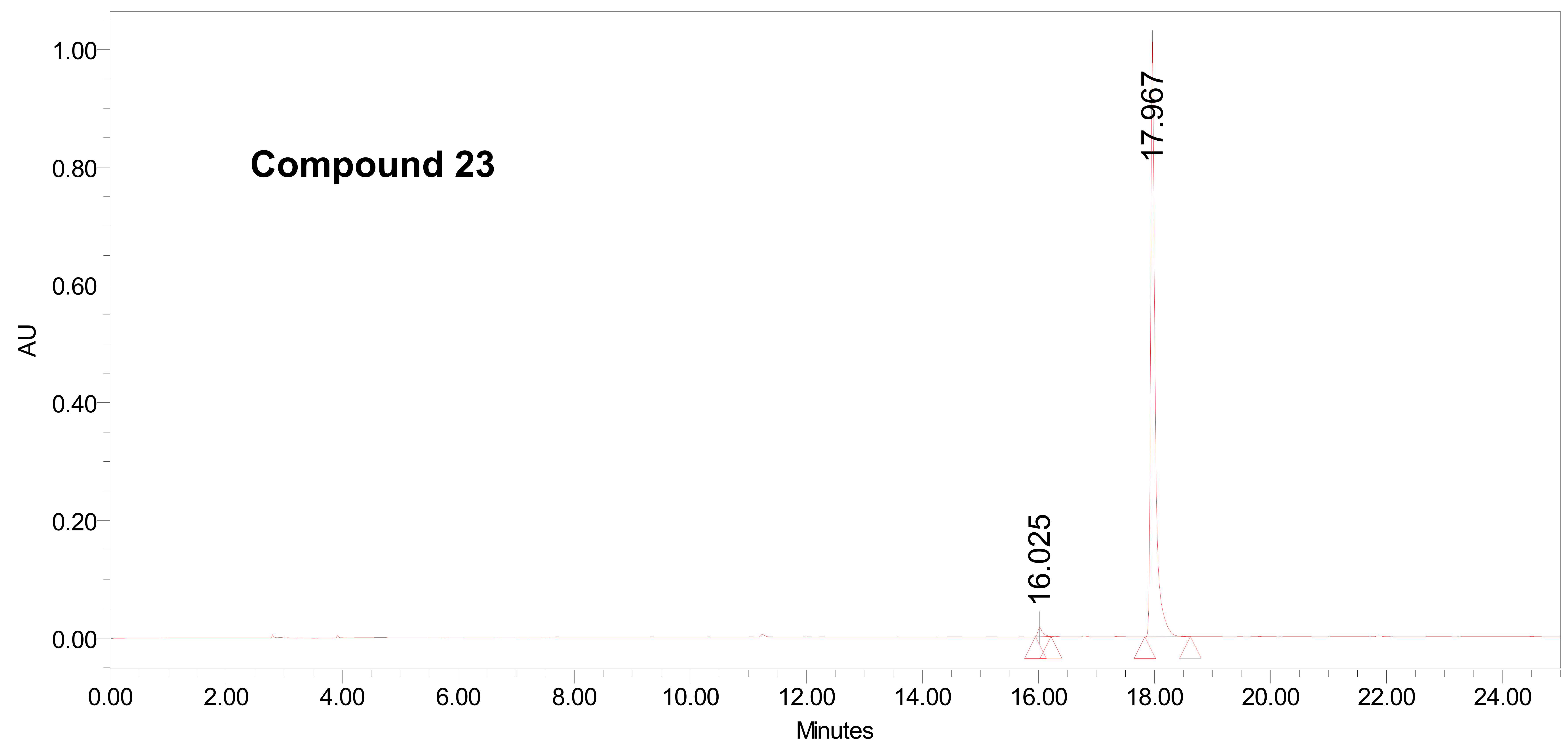

\begin{tabular}{|l|c|r|r|r|}
\multicolumn{5}{c|}{ Peak Results } \\
\begin{tabular}{|l|c|r|r|r|}
\hline & RT & Area & $\%$ Area & Height \\
\hline 1 & 16.025 & 86668 & 1.57 & 15155 \\
\hline 2 & 17.967 & 5437281 & 98.43 & 985651 \\
\hline
\end{tabular}
\end{tabular}

Reported by User: walkup Report Method: WalkUpReport Report Method ID 1098

Page: 1 of 1
Project Name: MedChemlMedChem2017-04

Date Printed:

$7 / 26 / 2019$

1:54:44 PM America/New_York 


\begin{tabular}{|llll|}
\hline & \multicolumn{1}{c|}{ S A M P E } & I N F O R M A T I O N \\
\hline \hline Sample Name: & 14125-014 & Acquired By: & walkup \\
Sample Type: & Unknown & Sample Set Name: & thuy20170417 \\
Vial: & 3 & Acq. Method Set: & MethodSetAB5T95B15Min \\
Injection \#: & 1 & Processing Method: & 220W280W15MIN \\
Injection Volume: & $10.00 \mathrm{ul}$ & Channel Name: & W2996 280.0nm-4.0 \\
Run Time: & 30.0 Minutes & Proc. Chnl. Descr.: & W2996 PDA 280.0 nm at 4.0 \\
& & & \\
Date Acquired: & $4 / 17 / 2017$ 11:36:11 AM EDT & & \\
Date Processed: & $7 / 26 / 2019$ 12:55:55 PM EDT & & \\
\hline
\end{tabular}

\section{Auto-Scaled Chromatogram}

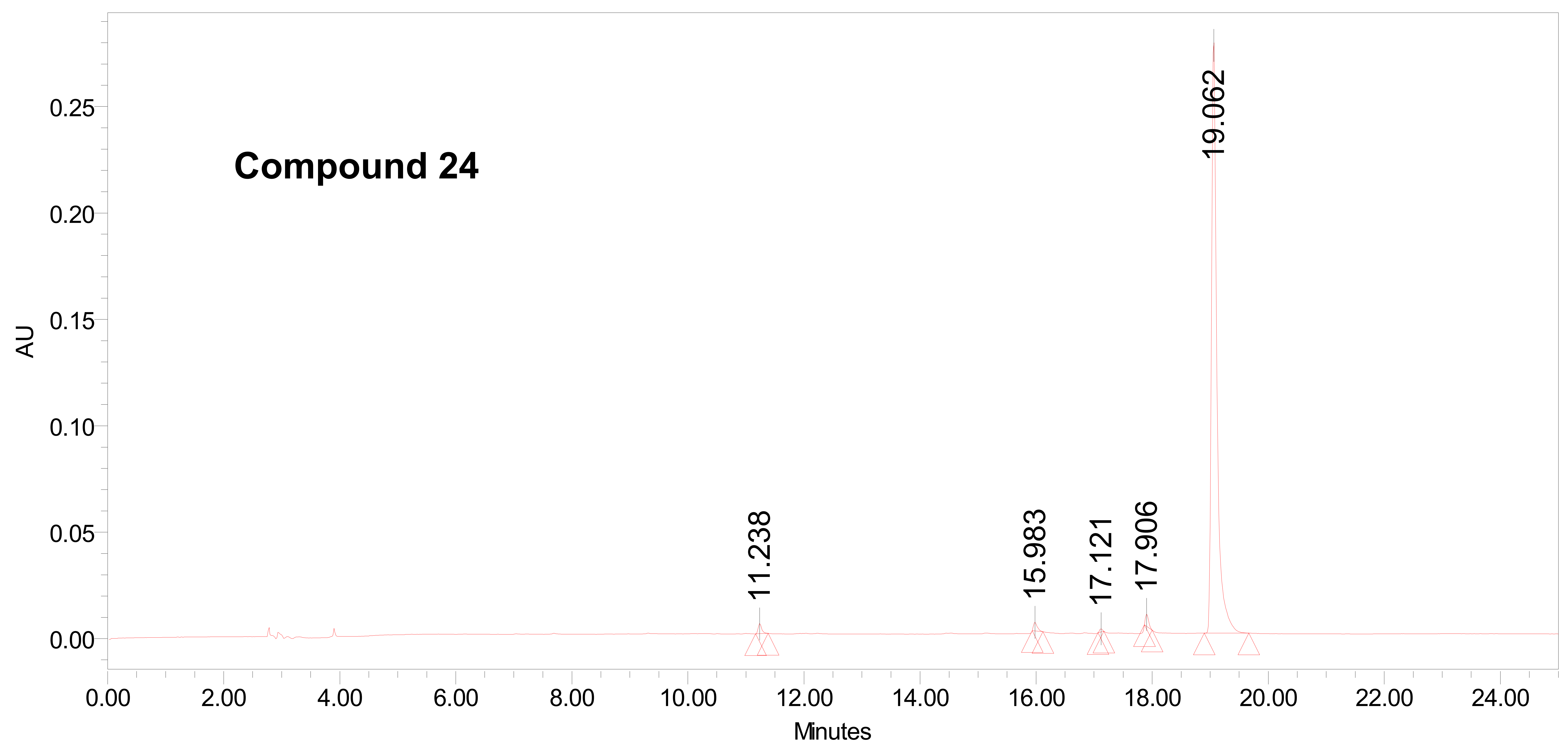

\begin{tabular}{|l|c|r|r|r|}
\multicolumn{5}{|c|}{ Peak Results } \\
\hline & RT & \multicolumn{1}{c|}{ Area } & \% Area & Height \\
\hline 1 & 11.238 & 20029 & 1.06 & 4590 \\
\hline 2 & 15.983 & 18543 & 0.99 & 4065 \\
\hline 3 & 17.121 & 5046 & 0.27 & 1471 \\
\hline 4 & 17.906 & 22046 & 1.17 & 5750 \\
\hline 5 & 19.062 & 1815646 & 96.51 & 276871 \\
\hline
\end{tabular}

Reported by User: walkup

Report Method: WalkUpReport

Report Method ID 1098

Page: 1 of 1
Project Name: MedChemlMedChem2017-04

Date Printed:

$7 / 26 / 2019$

12:56:17 PM America/New_York 


\begin{tabular}{|llll|}
\hline & \multicolumn{1}{c|}{ S A M P E } & I N F O R M A T I O N \\
\hline \hline Sample Name: & $14125-073$ & Acquired By: & walkup \\
Sample Type: & Unknown & Sample Set Name: & thuy20170518 \\
Vial: & 26 & Acq. Method Set: & MethodSetAB5T95B15Min \\
Injection \#: & 1 & Processing Method: & 220W280W15MIN \\
Injection Volume: & $10.00 \mathrm{ul}$ & Channel Name: & W2996 280.0nm-4.0 \\
Run Time: & 30.0 Minutes & Proc. Chnl. Descr.: & W2996 PDA 280.0 nm at 4.0 \\
& & & \\
Date Acquired: & $5 / 18 / 20171: 41: 42$ PM EDT & & \\
Date Processed: & $7 / 26 / 20191: 40: 38$ PM EDT & & \\
\hline
\end{tabular}

\section{Auto-Scaled Chromatogram}

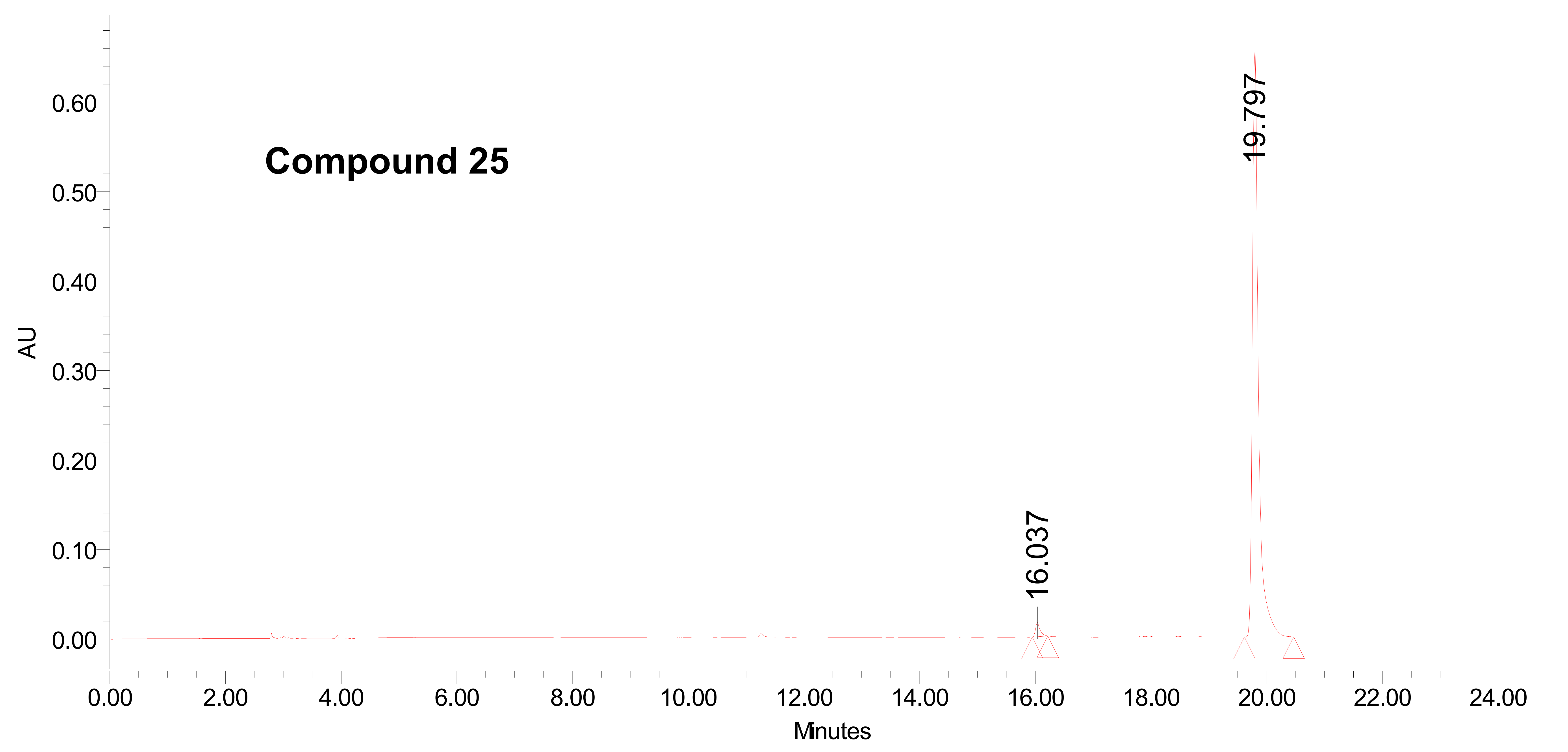

\begin{tabular}{|l|c|r|r|r|}
\multicolumn{5}{c|}{ Peak Results } \\
\begin{tabular}{|c|c|r|r|r|}
\hline & RT & Area & $\%$ Area & Height \\
\hline 1 & 16.037 & 86274 & 1.77 & 15488 \\
\hline 2 & 19.797 & 4799549 & 98.23 & 658698 \\
\hline
\end{tabular}
\end{tabular}

Reported by User: walkup Report Method: WalkUpReport Report Method ID 1022

Page: 1 of 1
Project Name: MedChemlMedChem2017-05

Date Printed:

7/26/2019

1:40:47 PM America/New_York 


\begin{tabular}{|llll|}
\hline & \multicolumn{1}{c|}{ S A M P E } & I N F O R M A T I O N \\
\hline \hline Sample Name: & $14125-085$ & Acquired By: & walkup \\
Sample Type: & Unknown & Sample Set Name: & thuy20170524 \\
Vial: & 21 & Acq. Method Set: & MethodSetAB5T95B15Min \\
Injection \#: & 1 & Processing Method: & 220W280W15MIN \\
Injection Volume: & $10.00 \mathrm{ul}$ & Channel Name: & W2996 280.0nm-4.0 \\
Run Time: & 30.0 Minutes & Proc. Chnl. Descr.: & W2996 PDA 280.0 nm at 4.0 \\
& & & \\
Date Acquired: & $5 / 24 / 2017$ 10:19:32 AM EDT & & \\
Date Processed: & $7 / 26 / 2019$ 12:59:59 PM EDT & & \\
\hline
\end{tabular}

\section{Auto-Scaled Chromatogram}

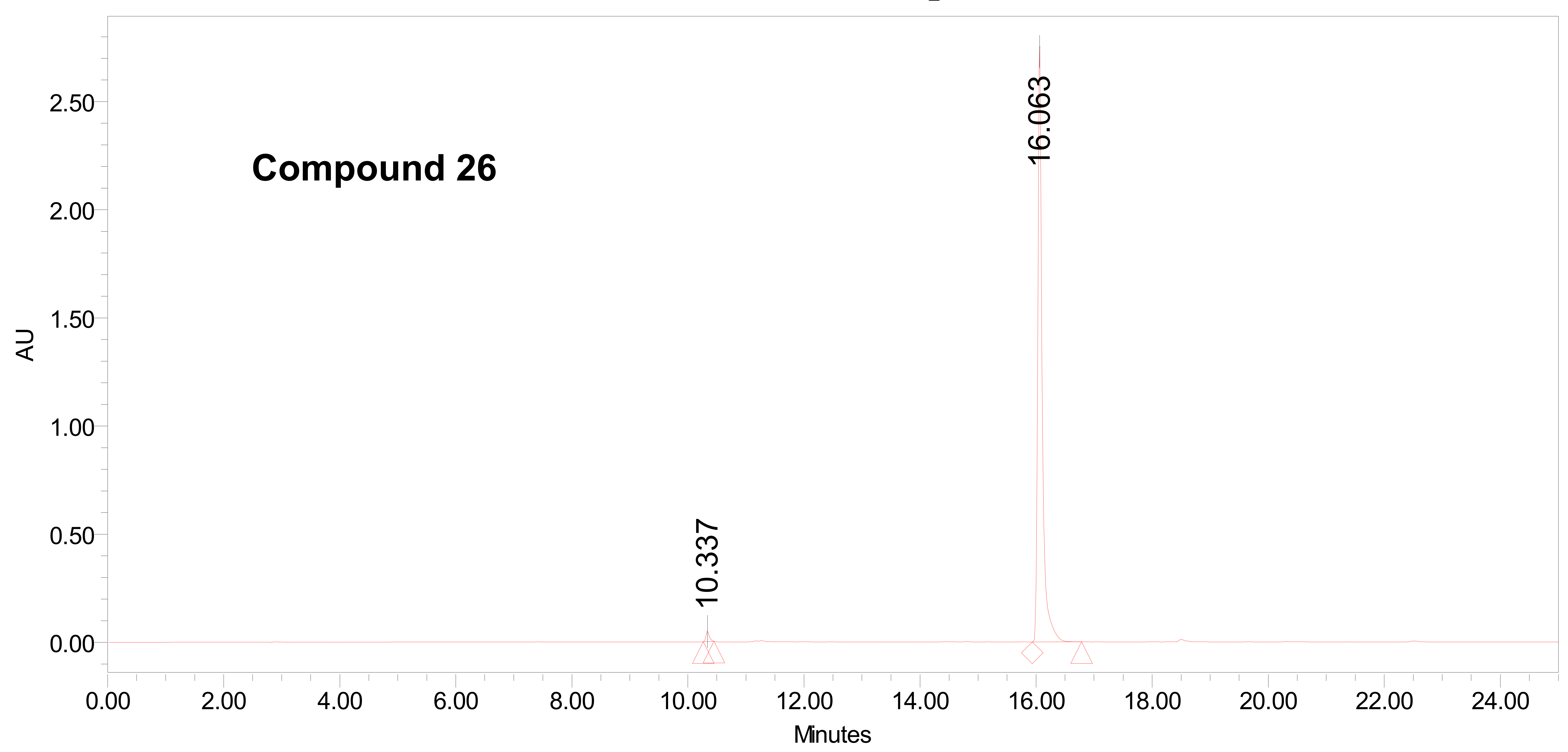

\begin{tabular}{|l|c|r|r|r|}
\multicolumn{5}{c|}{ Peak Results } \\
\hline & RT & Area & $\%$ Area & Height \\
\hline 1 & 10.337 & 200676 & 1.34 & 47142 \\
\hline 2 & 16.063 & 14758017 & 98.66 & 2709074 \\
\hline
\end{tabular}

Reported by User: walkup Report Method: WalkUpReport Report Method ID 1022

Page: 1 of 1
Project Name: MedChemlMedChem2017-05

Date Printed:

$7 / 26 / 2019$

1:00:25 PM America/New_York 


\begin{tabular}{|llll|}
\hline & \multicolumn{2}{c|}{ S A M P LE } & I N F O R M A T I O N \\
\hline \hline Sample Name: & 14125-086 & Acquired By: & walkup \\
Sample Type: & Unknown & Sample Set Name: & thuy20170526 \\
Vial: & 90 & Acq. Method Set: & MethodSetAB5T95B15Min \\
Injection \#: & 1 & Processing Method & 220W280W15MIN \\
Injection Volume: & 10.00 ul & Channel Name: & W2996 220.0nm-2.0 \\
Run Time: & 30.0 Minutes & Proc. Chnl. Descr.: W2996 PDA 220.0 nm at 2.0 \\
& & & \\
Date Acquired: & $5 / 26 / 201712: 25: 08$ PM EDT & & \\
Date Processed: & $5 / 26 / 201712: 55: 20$ PM EDT & & \\
\hline
\end{tabular}

\section{Auto-Scaled Chromatogram}

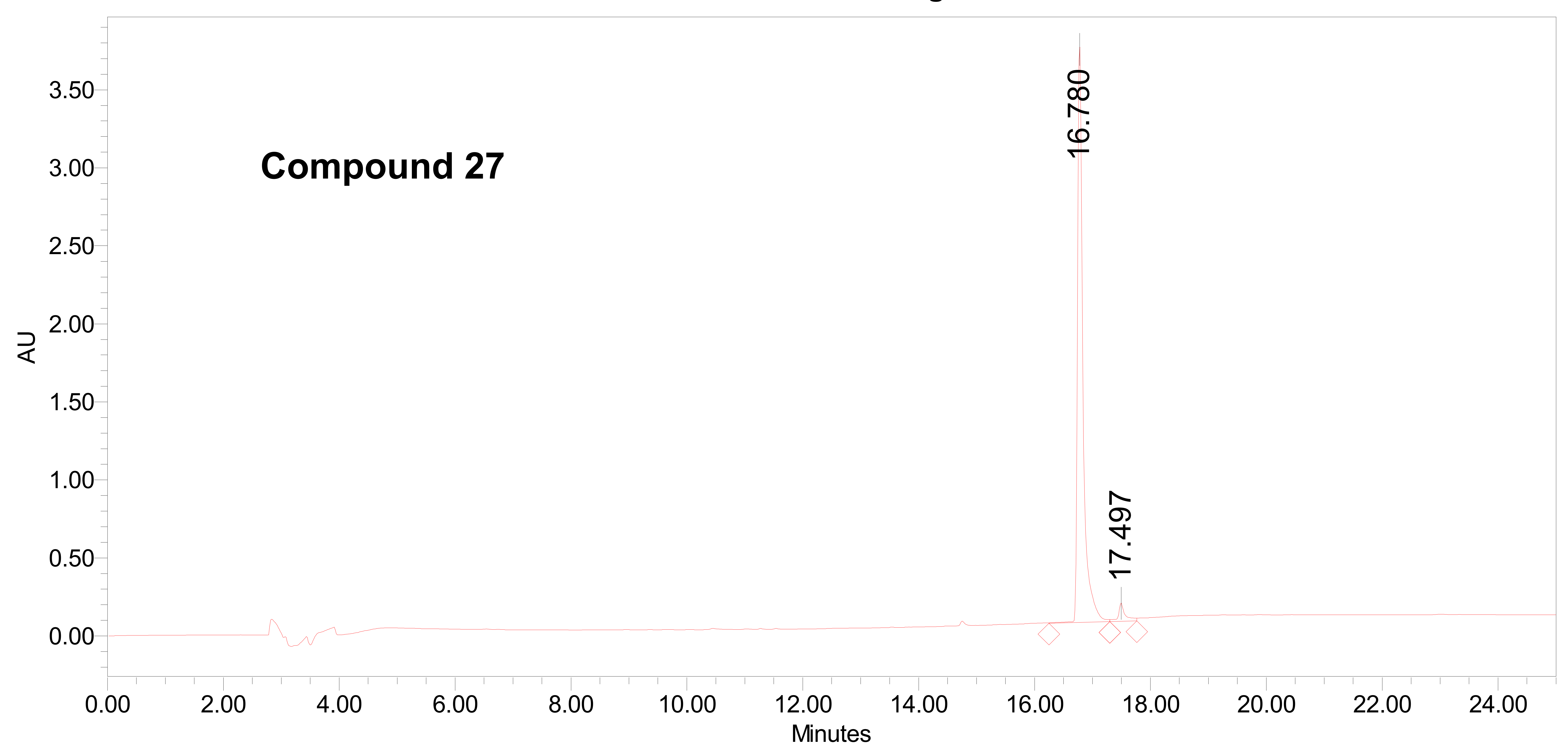

\begin{tabular}{|r|c|r|r|r|}
\multicolumn{5}{|c|}{ Peak Results } \\
\hline & RT & \multicolumn{1}{|c|}{ Area } & $\%$ Area & Height \\
\hline 1 & 16.780 & 24449045 & 96.20 & 3713653 \\
\hline 2 & 17.497 & 967071 & 3.80 & 114803 \\
\hline
\end{tabular}




\begin{tabular}{|llll|}
\hline & \multicolumn{1}{c|}{ S A M P E } & I N F O R M A T I O N \\
\hline \hline Sample Name: & $14200-087$ & Acquired By: & walkup \\
Sample Type: & Unknown & Sample Set Name: & thuy20171121a \\
Vial: & 1 & Acq. Method Set: & MethodSetAB5T95B15Min \\
Injection \#: & 1 & Processing Method: & 220W280W15MIN \\
Injection Volume: & $20.00 \mathrm{ul}$ & Channel Name: & W2996 280.0nm-4.0 \\
Run Time: & 30.0 Minutes & Proc. Chnl. Descr.: & W2996 PDA 280.0 nm at 4.0 \\
& & & \\
Date Acquired: & $11 / 21 / 201712: 33: 19$ PM EST & & \\
Date Processed: & $7 / 26 / 20191: 20: 10$ PM EDT & & \\
\hline
\end{tabular}

\section{Auto-Scaled Chromatogram}

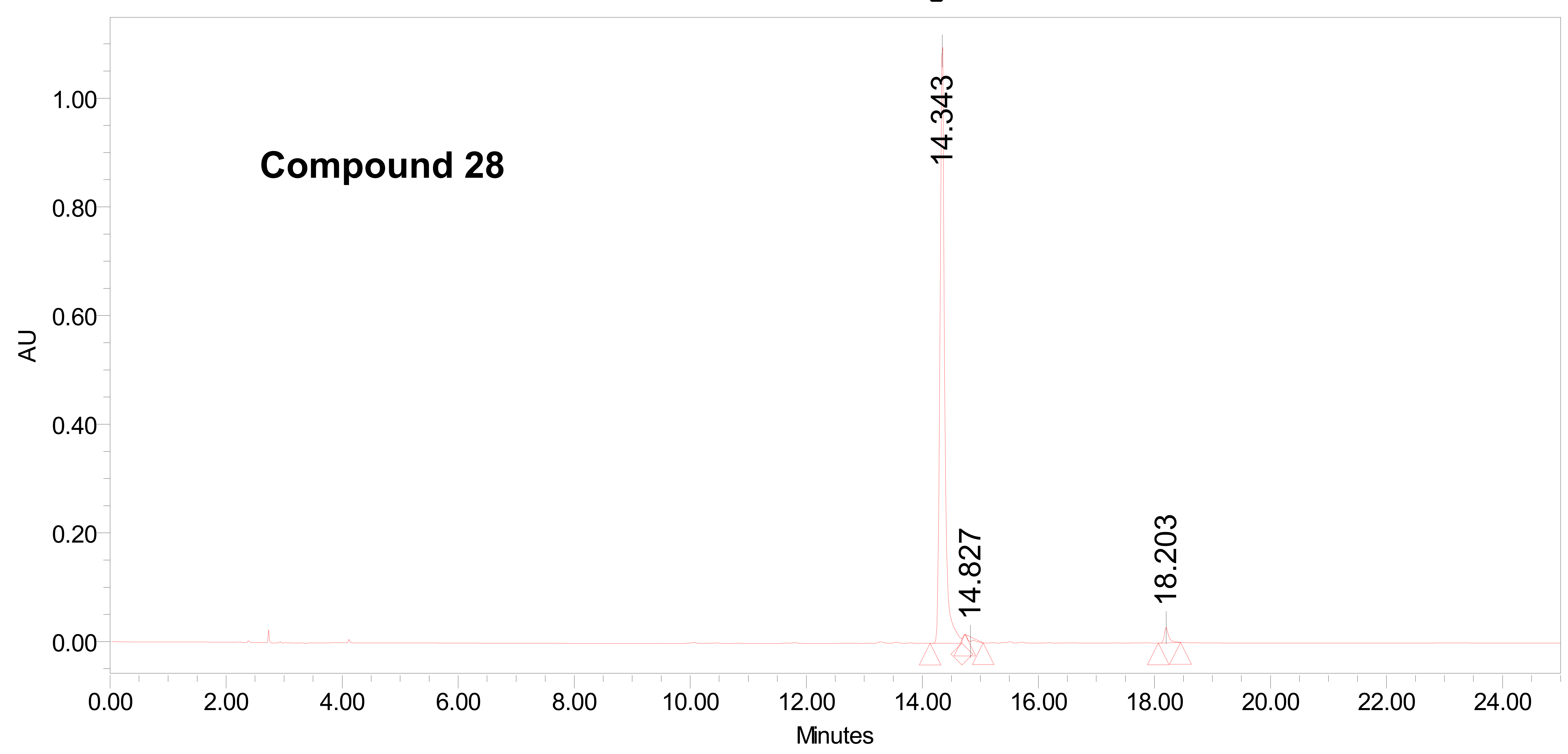

\begin{tabular}{|l|c|r|r|r|}
\multicolumn{5}{|c|}{ Peak Results } \\
\begin{tabular}{|r|r|r|r|r|}
\hline & RT & Area & $\%$ Area & Height \\
\hline 1 & 14.343 & 6243027 & 96.64 & 1089955 \\
\hline 2 & 14.827 & 69255 & 1.07 & -8700 \\
\hline 3 & 18.203 & 148083 & 2.29 & 27988 \\
\hline
\end{tabular}
\end{tabular}

Reported by User: walkup Report Method: WalkUpReport Report Method ID 1022

Page: 1 of 1
Project Name: $\quad$ MedChemlMedChem2017-11

Date Printed:

$7 / 26 / 2019$

1:20:58 PM America/New_York 


\begin{tabular}{|llll|}
\hline & \multicolumn{1}{c|}{ S A M P L E } & I N F O R M A T I O N \\
\hline \hline Sample Name: & $14125-045$ & Acquired By: & walkup \\
Sample Type: & Unknown & Sample Set Name: & thuy20170502 \\
Vial: & 110 & Acq. Method Set: & MethodSetAB5T95B15Min \\
Injection \#: & 1 & Processing Method: & 220W280W15MIN \\
Injection Volume: & $10.00 \mathrm{ul}$ & Channel Name: & W2996 280.0nm-4.0 \\
Run Time: & 30.0 Minutes & Proc. Chnl. Descr.: & W2996 PDA 280.0 nm at 4.0 \\
& & & \\
Date Acquired: & $5 / 2 / 201711: 34: 25$ AM EDT & & \\
Date Processed: & $7 / 26 / 20191: 03: 58$ PM EDT & & \\
\hline
\end{tabular}

\section{Auto-Scaled Chromatogram}

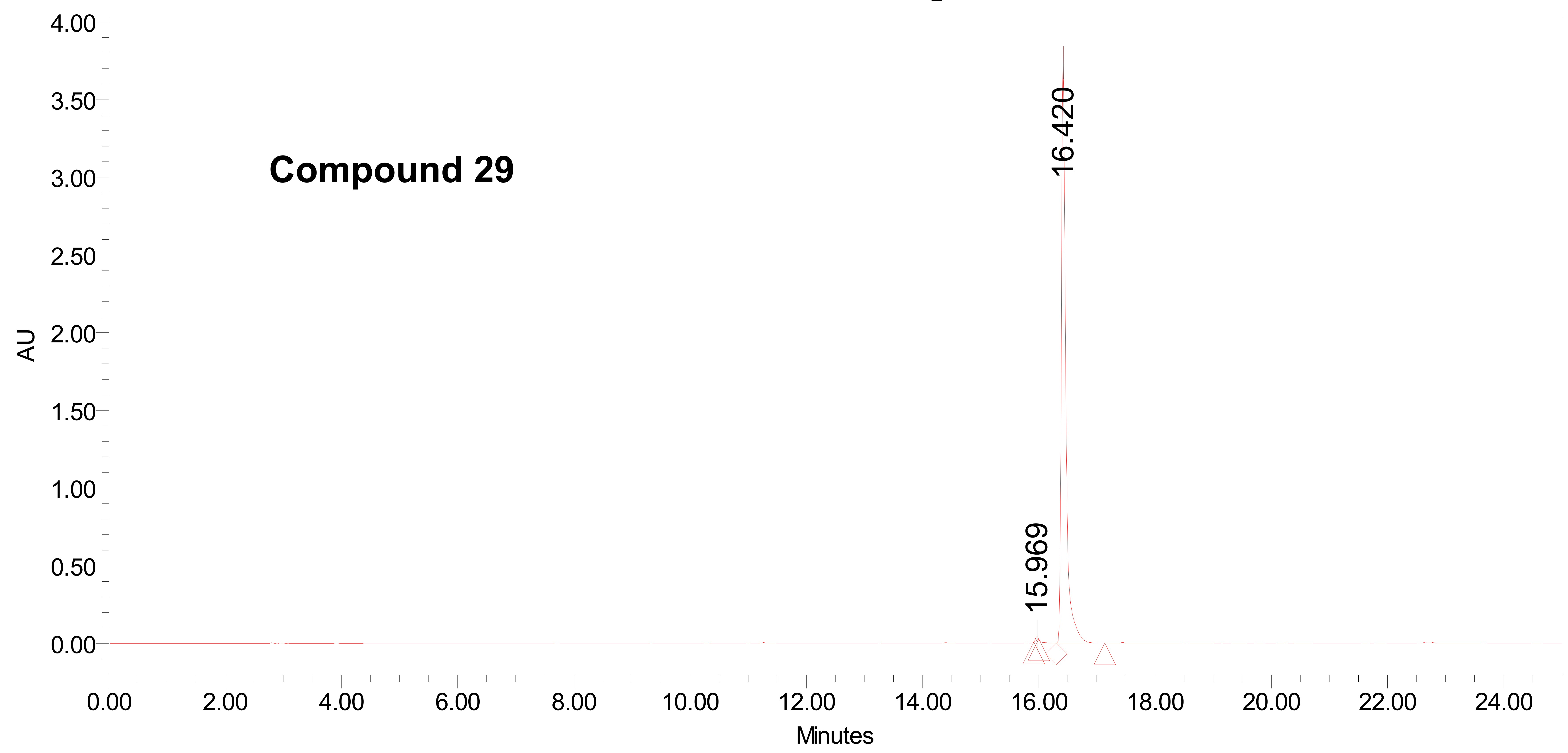

\begin{tabular}{|l|c|r|r|r|}
\multicolumn{5}{|c|}{ Peak Results } \\
\hline & RT & Area & $\%$ Area & Height \\
\hline 1 & 15.969 & 73182 & 0.36 & 24470 \\
\hline 2 & 16.420 & 20095176 & 99.64 & 3744914 \\
\hline
\end{tabular}

Reported by User: walkup Report Method: WalkUpReport Report Method ID 1022

Page: 1 of 1
Project Name: MedChemlMedChem2017-05

Date Printed:

$7 / 26 / 2019$

1:04:49 PM America/New_York 


\begin{tabular}{|llll|}
\hline & \multicolumn{1}{c|}{ S A M P E } & I N F O R M A T I O N \\
\hline \hline Sample Name: & $14125-049$ & Acquired By: & walkup \\
Sample Type: & Unknown & Sample Set Name: & thuy20170504 \\
Vial: & 40 & Acq. Method Set: & MethodSetAB5T95B15Min \\
Injection \#: & 1 & Processing Method: & 220W280W15MIN \\
Injection Volume: & $10.00 \mathrm{ul}$ & Channel Name: & W2996 280.0nm-4.0 \\
Run Time: & 30.0 Minutes & Proc. Chnl. Descr.: & W2996 PDA 280.0 nm at 4.0 \\
& & & \\
Date Acquired: & $5 / 4 / 20179: 56: 58$ AM EDT & & \\
Date Processed: & $7 / 26 / 20191: 13: 31$ PM EDT & & \\
\hline
\end{tabular}

\section{Auto-Scaled Chromatogram}

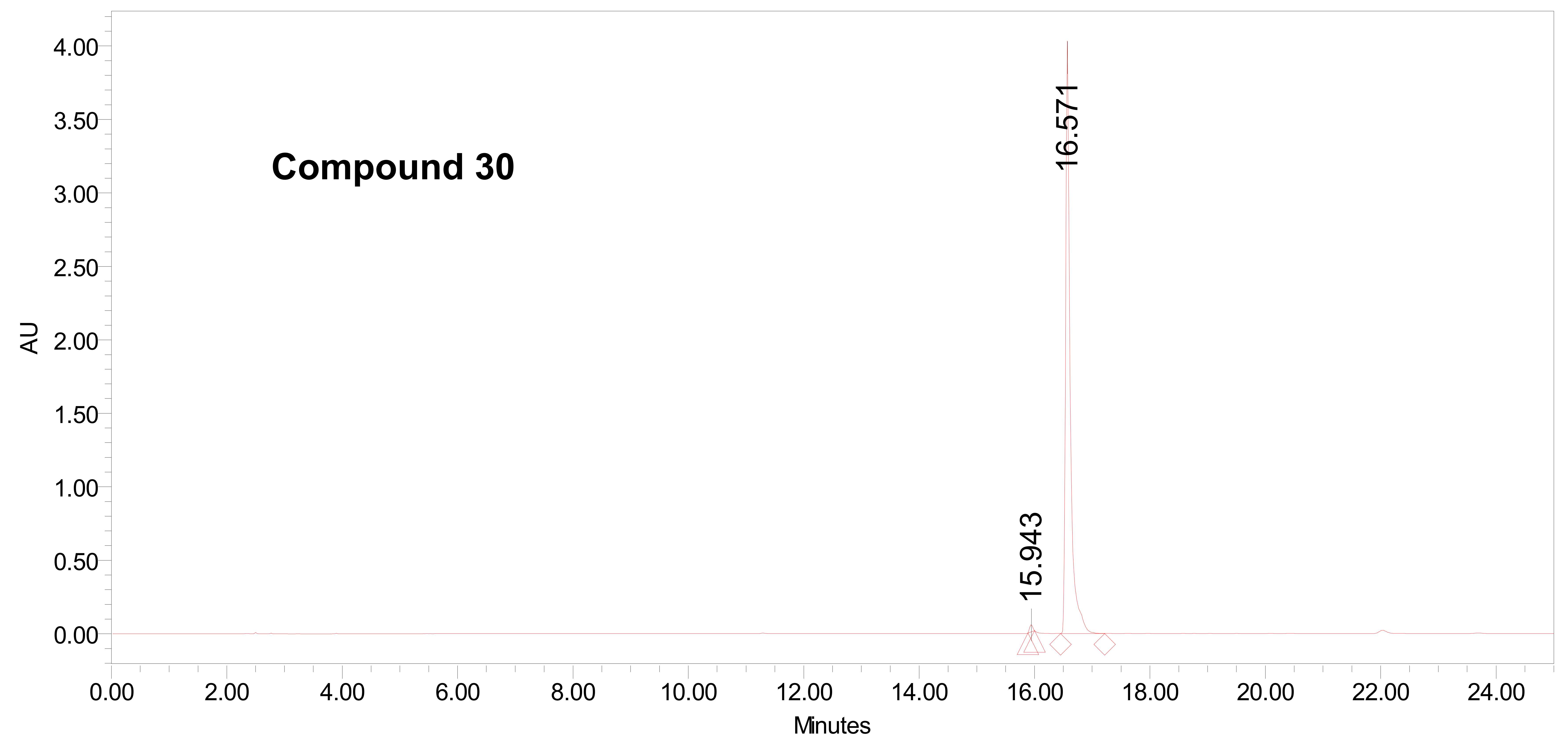

\begin{tabular}{|l|c|c|r|r|}
\multicolumn{5}{|c}{ Peak Results } \\
\hline & RT & Area & $\%$ Area & Height \\
\hline 1 & 15.943 & 176307 & 0.79 & 46643 \\
\hline 2 & 16.571 & 22215043 & 99.21 & 3950631 \\
\hline
\end{tabular}

Reported by User: walkup Report Method: WalkUpReport Report Method ID 1022

Page: 1 of 1
Project Name: MedChemlMedChem2017-05

Date Printed:

7/26/2019

1:14:04 PM America/New_York 


\begin{tabular}{|llll|}
\hline & \multicolumn{1}{c|}{ S A M P E } & I N F O R M A T I O N \\
\hline \hline Sample Name: & $14125-050$ & Acquired By: & walkup \\
Sample Type: & Unknown & Sample Set Name: & thuy20170504 \\
Vial: & 41 & Acq. Method Set: & MethodSetAB5T95B15Min \\
Injection \#: & 1 & Processing Method: & 220W280W15MIN \\
Injection Volume: & $10.00 \mathrm{ul}$ & Channel Name: & W2996 280.0nm-4.0 \\
Run Time: & 30.0 Minutes & Proc. Chnl. Descr.: & W2996 PDA 280.0 nm at 4.0 \\
& & & \\
Date Acquired: & $5 / 4 / 201710: 27: 58$ AM EDT & & \\
Date Processed: & $7 / 26 / 20191: 09: 10$ PM EDT & & \\
\hline
\end{tabular}

\section{Auto-Scaled Chromatogram}

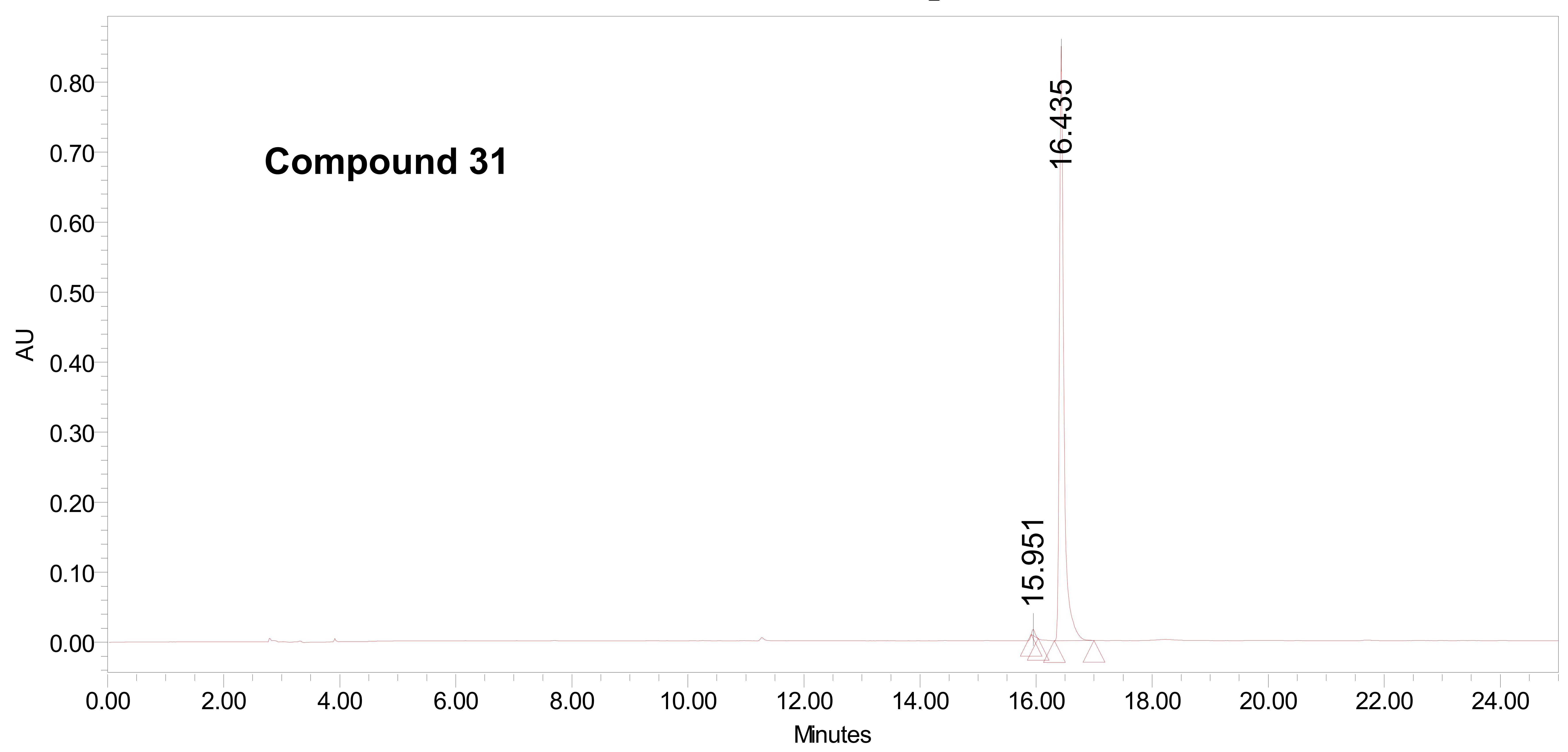

\begin{tabular}{|l|c|r|r|r|}
\multicolumn{5}{c|}{ Peak Results } \\
\begin{tabular}{|l|c|r|r|r|}
\hline & RT & Area & $\%$ Area & Height \\
\hline 1 & 15.951 & 30369 & 0.68 & 8701 \\
\hline 2 & 16.435 & 4429181 & 99.32 & 825952 \\
\hline
\end{tabular}
\end{tabular}

Reported by User: walkup Report Method: WalkUpReport Report Method ID 1022

Page: 1 of 1
Project Name: MedChemlMedChem2017-05

Date Printed:

$7 / 26 / 2019$

1:12:04 PM America/New_York 


\begin{tabular}{|llll|}
\hline & \multicolumn{1}{c|}{ S A M P E } & I N F O R M A T I O N \\
\hline \hline Sample Name: & $14125-067$ & Acquired By: & walkup \\
Sample Type: & Unknown & Sample Set Name: & thuy20170516 \\
Vial: & 101 & Acq. Method Set: & MethodSetAB5T95B15Min \\
Injection \#: & 1 & Processing Method: & 220W280W15MIN \\
Injection Volume: & $10.00 \mathrm{ul}$ & Channel Name: & W2996 280.0nm-4.0 \\
Run Time: & 30.0 Minutes & Proc. Chnl. Descr.: & W2996 PDA 280.0 nm at 4.0 \\
& & & \\
Date Acquired: & $5 / 16 / 2017$ 12:22:38 PM EDT & & \\
Date Processed: & $7 / 26 / 2019$ 1:15:14 PM EDT & & \\
\hline
\end{tabular}

\section{Auto-Scaled Chromatogram}

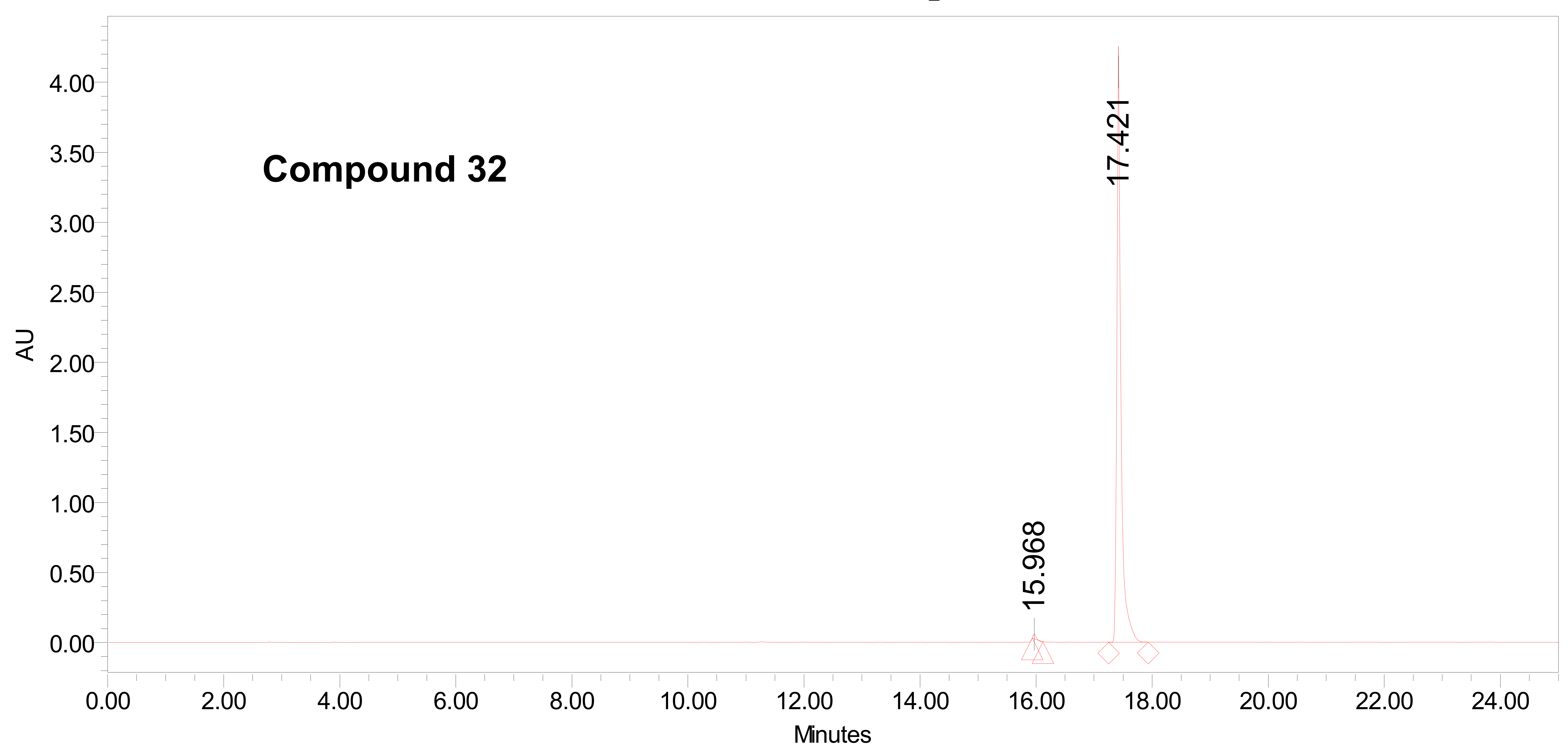

\begin{tabular}{|l|c|c|r|r|}
\multicolumn{5}{|c|}{ Peak Results } \\
\begin{tabular}{|c|c|r|r|r|}
\hline & RT & Area & $\%$ Area & Height \\
\hline 1 & 15.968 & 114549 & 0.55 & 32040 \\
\hline 2 & 17.421 & 20783322 & 99.45 & 4002212 \\
\hline
\end{tabular}
\end{tabular}

Reported by User: walkup Report Method: WalkUpReport Report Method ID 1022

Page: 1 of 1
Project Name: MedChemlMedChem2017-05

Date Printed:

$7 / 26 / 2019$

1:15:53 PM America/New_York 


\begin{tabular}{|llll|}
\hline & \multicolumn{1}{c|}{ S A M P E } & I N F O R M A T I O N \\
\hline \hline Sample Name: & $14125-078$ & Acquired By: & walkup \\
Sample Type: & Unknown & Sample Set Name: & thuy20170519 \\
Vial: & 30 & Acq. Method Set: & MethodSetAB5T95B15Min \\
Injection \#: & 1 & Processing Method: & 220W280W15MIN \\
Injection Volume: & $10.00 \mathrm{ul}$ & Channel Name: & W2996 280.0nm-4.0 \\
Run Time: & 30.0 Minutes & Proc. Chnl. Descr.: & W2996 PDA 280.0 nm at 4.0 \\
& & & \\
Date Acquired: & $5 / 19 / 2017$ 1:07:31 PM EDT & & \\
Date Processed: & $7 / 26 / 20191: 41: 34$ PM EDT & & \\
\hline
\end{tabular}

\section{Auto-Scaled Chromatogram}

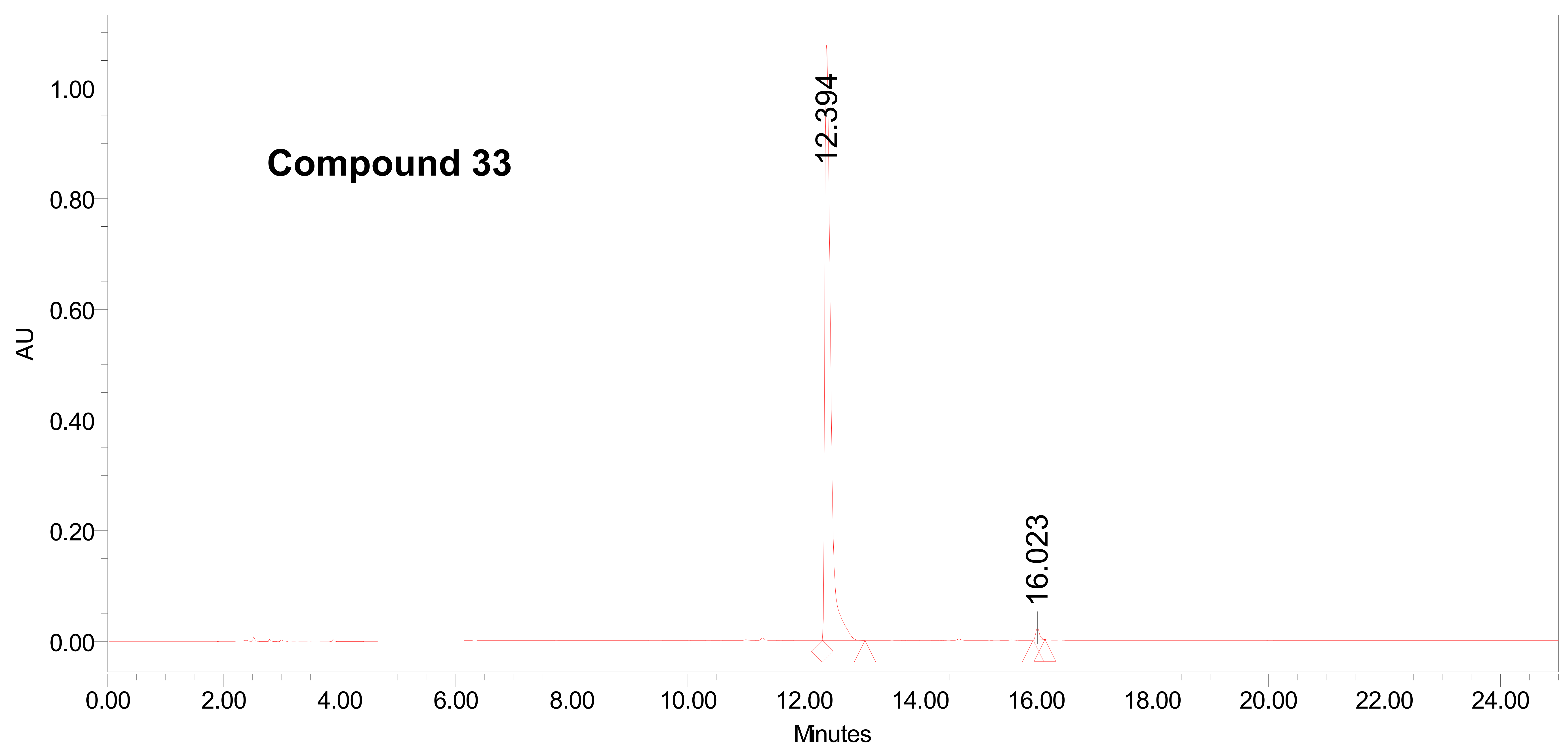

\begin{tabular}{|l|c|r|r|r|}
\multicolumn{5}{|c|}{ Peak Results } \\
\begin{tabular}{|r|r|r|r|r|}
\hline & RT & Area & $\%$ Area & Height \\
\hline 1 & 12.394 & 7590283 & 98.70 & 1117606 \\
\hline 2 & 16.023 & 100066 & 1.30 & 22000 \\
\hline
\end{tabular}
\end{tabular}

Reported by User: walkup Report Method: WalkUpReport Report Method ID 1022

Page: 1 of 1
Project Name: MedChemlMedChem2017-05

Date Printed:

7/26/2019

1:41:43 PM America/New_York 


\begin{tabular}{|llll|}
\hline & \multicolumn{1}{c|}{ S A M P E } & I N F O R M A T I O N \\
\hline \hline Sample Name: & 14125-028-2 & Acquired By: & walkup \\
Sample Type: & Unknown & Sample Set Name: & thuy20170421a \\
Vial: & 50 & Acq. Method Set: & MethodSetAB5T95B15Min \\
Injection \#: & 1 & Processing Method: & 220W280W15MIN \\
Injection Volume: & $10.00 \mathrm{ul}$ & Channel Name: & W2996 280.0nm-4.0 \\
Run Time: & 30.0 Minutes & Proc. Chnl. Descr.: & W2996 PDA 280.0 nm at 4.0 \\
& & & \\
Date Acquired: & $4 / 21 / 20172: 30: 07$ PM EDT & & \\
Date Processed: & $7 / 26 / 2019$ 1:43:46 PM EDT & & \\
\hline
\end{tabular}

\section{Auto-Scaled Chromatogram}

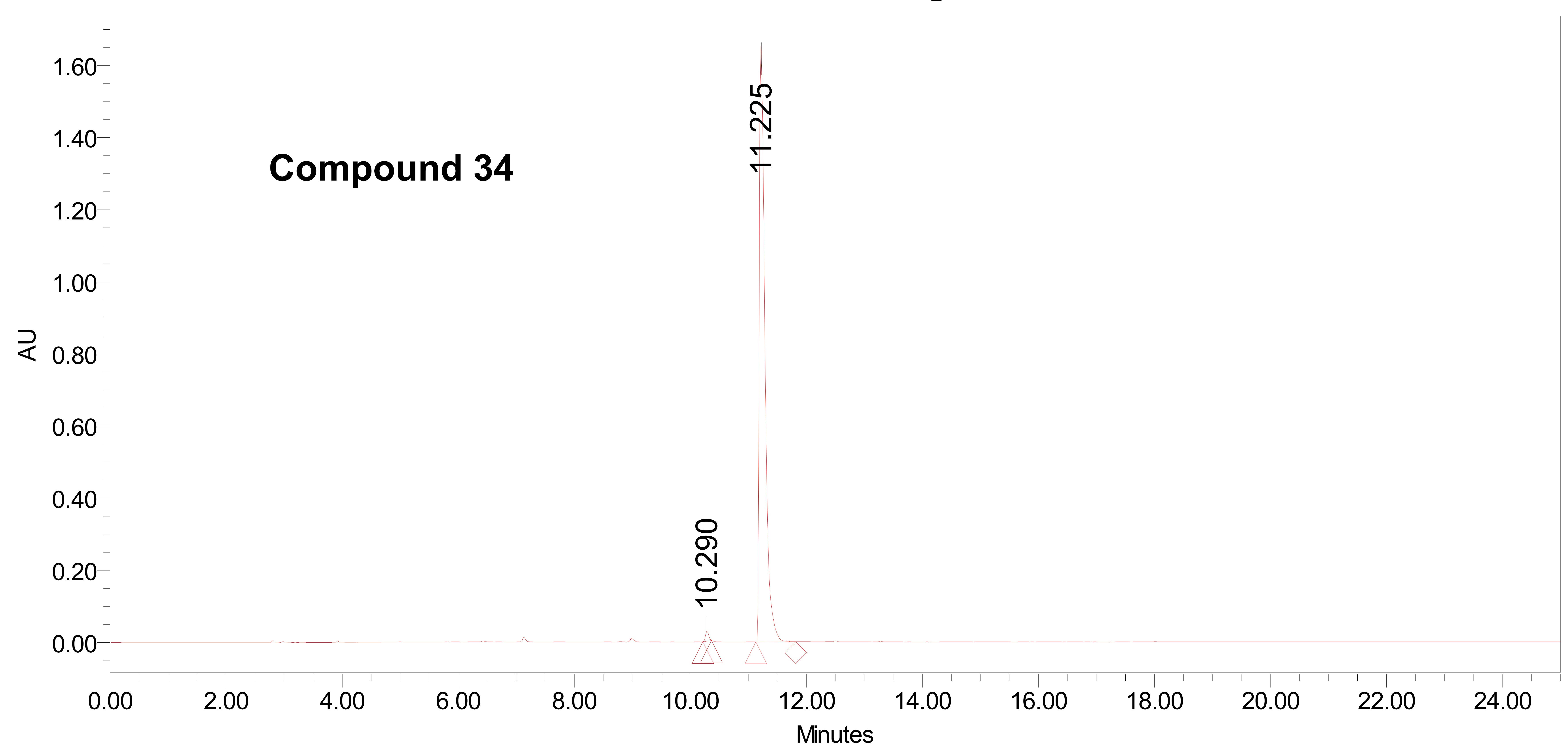

\begin{tabular}{|l|c|r|r|r|}
\multicolumn{5}{c|}{ Peak Results } \\
\hline & RT & Area & $\%$ Area & \multicolumn{1}{|c|}{ Height } \\
\hline 1 & 10.290 & 104084 & 0.91 & 26779 \\
\hline 2 & 11.225 & 11333640 & 99.09 & 1696802 \\
\hline
\end{tabular}

Reported by User: walkup Report Method: WalkUpReport Report Method ID 1098

Page: 1 of 1
Project Name: MedChemlMedChem2017-04

Date Printed:

$7 / 26 / 2019$

1:54:15 PM America/New_York 


\begin{tabular}{|llll|}
\hline & \multicolumn{1}{c|}{ S A M P LE } & I N F O R M A T I O N \\
\hline \hline Sample Name: & 14125-099 & Acquired By: & walkup \\
Sample Type: & Unknown & Sample Set Name: & thuy20170706 \\
Vial: & 20 & Acq. Method Set: & MethodSetAB5T95B15Min \\
Injection \#: & 1 & Processing Method & 220W280W15MIN \\
Injection Volume: & $10.00 \mathrm{ul}$ & Channel Name: & W2996 280.0nm-4.0 \\
Run Time: & 30.0 Minutes & Proc. Chnl. Descr.: & W2996 PDA 280.0 nm at 4.0 \\
& & & \\
Date Acquired: & $7 / 6 / 2017$ 9:39:15 AM EDT & & \\
Date Processed: & $7 / 6 / 201710: 09: 27$ AM EDT & & \\
\hline
\end{tabular}

\section{Auto-Scaled Chromatogram}

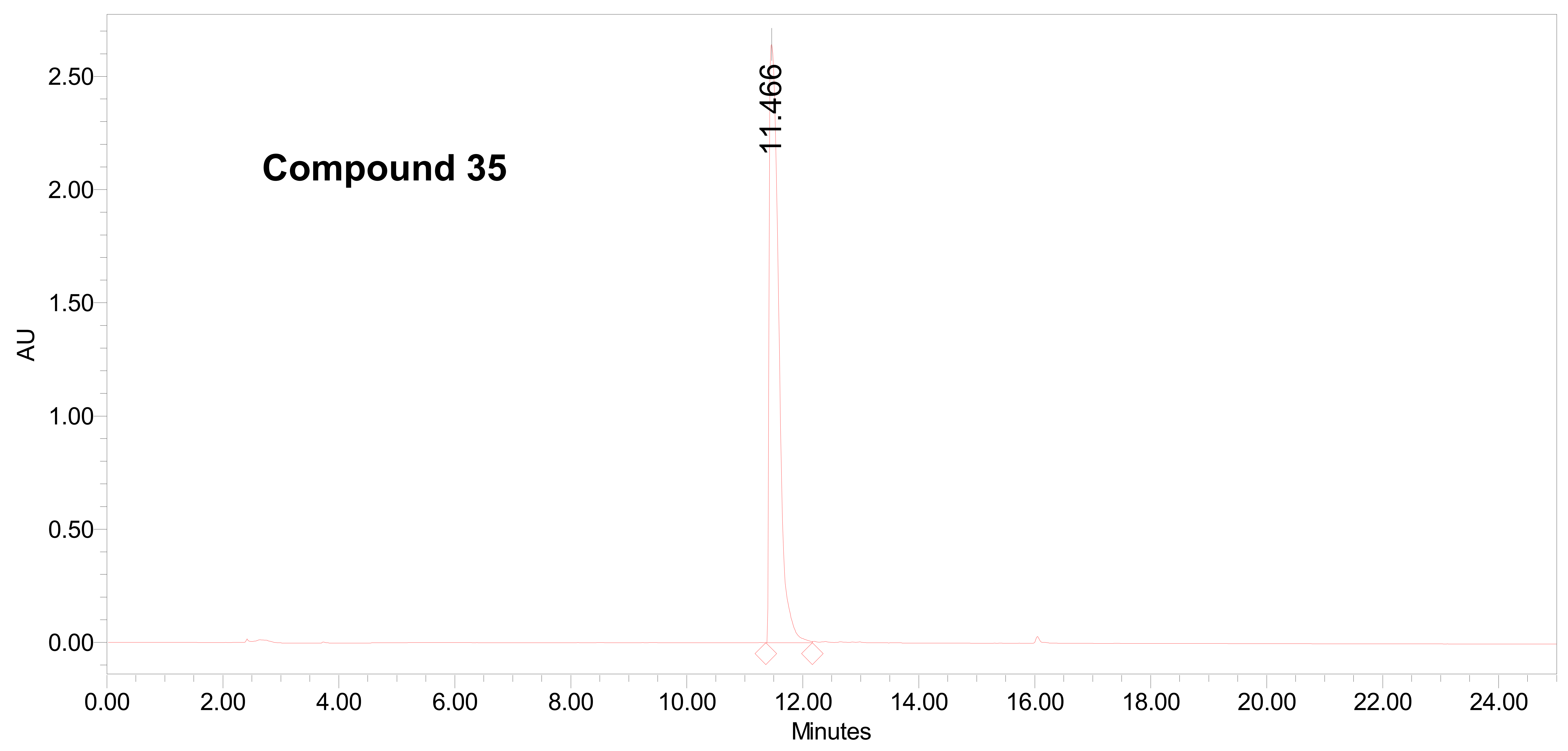

\begin{tabular}{|c|c|c|c|c|}
\multicolumn{5}{c}{ Peak Results } \\
\begin{tabular}{|c|c|c|c|c|}
\hline & RT & Area & $\%$ Area & Height \\
\hline 1 & 11.466 & 30664708 & 100.00 & 2649189 \\
\hline
\end{tabular}
\end{tabular}

